OPEN ACCESS

Edited by:

Senthil-Nathan Sengottayan,

Manonmaniam Sundaranar University,

India

Reviewed by:

Pin-Jun Wan

China National Rice Research Institute

(CAAS), China

Kai Lu,

Fujian Agriculture and Forestry

University, China

${ }^{*}$ Correspondence:

LinQuan $\mathrm{Ge}$

lage@yzu.edu.cn

QiSheng Song

songq@missouri.edu

${ }^{\dagger}$ Co-first authors

Specialty section:

This article was submitted to Invertebrate Physiology,

a section of the journal

Frontiers in Physiology

Received: 22 June 2019 Accepted: 03 September 2019 Published: 25 September 2019

Citation:

Ge L, Zhou Y, Gu H, Wu Q,

Zhou Z, Zheng S, Stanley $D$ and Song Q (2019) Male Selenoprotein

F-Like (SPF-L) Influences Female Reproduction and Population Growth in Nilaparvata lugens (Hemiptera:

Delphacidae).

Front. Physiol. 10:1196. doi: 10.3389/fphys.2019.01196

\section{Male Selenoprotein F-Like (SPF-L) Influences Female Reproduction and Population Growth in Nilaparvata lugens (Hemiptera: Delphacidae)}

\author{
LinQuan $\mathrm{Ge}^{1 *+}$, YongKai Zhou ${ }^{1+}$, HaoTian $\mathrm{Gu}^{1}$, Qing Wu ${ }^{1}$, Ze Zhou ${ }^{1}$, Sui Zheng ${ }^{1}$, \\ David Stanley² and QiSheng Song ${ }^{3 *}$
}

${ }^{1}$ School of Horticulture and Plant Protection, Yangzhou University, Yangzhou, China, ${ }^{2}$ Biological Control of Insects Research Laboratory, USDA/Agricultural Research Service, Columbia, MO, United States, ${ }^{3}$ Division of Plant Sciences, University of Missouri, Columbia, MO, United States

Selenoproteins serve in anti-oxidant and cellular redox functions in almost all organisms. A recent study characterized a selenoprotein F-like (SPF-L) in the brown plant hopper's $(\mathrm{BPH})$, Nilaparvata lugens, male accessory glands (MAGs), raised the question of whether the SPF-L is associated with female fecundity. In this study, SPF-L mRNA was found to be enriched in the internal reproductive organ $(\mathrm{IRO})$ of virgin males, also expressed relatively stably in virgin males and females, and dietary dsSPF-L-treatments led to reduced MAG protein and Arginine content. Knockdown of NISPF- $L$ in unmated males did not influence juvenile hormone $(\mathrm{JH})$ III and ecdysteroid titers, however, dsSPF-L-treated mated males had increased JH III titer, and reduced ecdysteroid titer compared to controls. After mating with dsSPF-L-treated males, female partners had reduced fat body and ovary soluble proteins and $\mathrm{JH}$ III tier and vitellogenin (Vg) mRNA levels, but no alterations in ecdysteroid titer, body weight or longevity. The experimental females had prolonged pre-oviposition periods and they laid fewer eggs, which suffered reduced hatching rates and population growth index (PGI). Such mating also led to impaired IRO development in males and females, which was confirmed by immunofluorescence staining. We infer that SPF-L affects reproductive success of males and their partners.

Keywords: Nilaparvata lugens, selenoprotein, seminal fluids, mating, fecundity

\section{INTRODUCTION}

A great diversity of reproductive strategies have evolved in sexual organisms. Aside from sperm transfer, male seminal fluids have a role in post-copulatory reproductive success because they are involved in the fertilization processes and sexual selection in animals (Poinani, 2006). In some male insects, the seminal fluids, secreted mainly by accessory glands, contribute to the formation of a mating plug that prevents re-mating or sperm efflux. Seminal fluids protect sperm and alter female behavior and physiology. In particular, in some species, it leads to rejecting subsequent males and facilitating feeding, ovulation, egg production and longevity (Simmons, 2001; Ravi Ram and Wolfner, 2007; Avila et al., 2011; Clifton et al., 2014). Some proteins responsible for these events have been identified from the accessory glands of Drosophila melanogaster (Ravi Ram and Wolfner, 2007, 2009). 
The brown planthopper (BPH), Nilaparvata lugens Stål (Hemiptera: Delphacidae) is a serious rice pest insect in Asia (Cheng, 2014), with a history of devastating outbreaks. Recent advances, such as new BPH-resistant rice varieties, new insecticides and integrated pest management programs help mitigate $\mathrm{BPH}$ damage. Nonetheless, BPHs poses real threats to rice cropping systems because some agricultural chemicals, such as the insecticide triazophs (TZP) and the antibiotic jinggangmycin (JGM), stimulate reproduction and population increases (Ge et al., 2010a; Wang et al., 2010). One of the molecular mechanisms of the increased reproduction is increasing male accessory gland protein (MAGP) content in males and increased MAGP transfer to females. The increased MAGP transfer leads to enhanced egg laying (Long et al., 2010) and almost life-long refractoriness to further insemination. $\mathrm{Yu}$ et al. (2016) used proteomic analyses to characterize the seminal fluid proteins composition in $\mathrm{BPH}$ male and some new examples were identified in male internal reproductive organs (IROs), and transferred to females via copulation, including a selenoprotein F-like (SPF-L), epidermal growth factor domain-containing proteins and a neuropeptide ion transport-like peptide. We suspect these and other transferred proteins are responsible for increased egg production and deposition in females.

Selenoproteins in human seminal fluid protect sperm during storage (Michaelis et al., 2014). Nevertheless, the roles of SPF-L in male BPH fertility and how SPF-L modulate female fecundity after copulation remain to be addressed. These proteins exist in almost all life forms. They feature the specialized amino acid selenocysteine in their reactive center, and mainly function as redox-enzymes (Stadtman, 1996). Selenium is essential for male fertility in mammals (Brown and Burk, 1973). Selenium deficiency leads to impaired sperm motility and flagellum loss (Brown and Burk, 1973), and diets lacking it result in shorter life spans (Martin-Romero et al., 2001).

Selenium is likely an essential micronutrient in all animals, which led to our hypothesis that male SPF-L influences male and female fitness, observed as an increase in offspring. Here, we report on the outcomes of experiments designed to test our hypothesis.

\section{MATERIALS AND METHODS}

\section{Rice Variety and Insects}

The rice (Oryza sativa L.) cultivar, Ningjing 4 (japonica rice), is commonly planted in Jiangsu Province, China. After soaking and germination, the seeds were sown in outdoor standard cement tanks (height $60 \mathrm{~cm}$, width $100 \mathrm{~cm}$, and length $200 \mathrm{~cm}$ ). The sixleaf seedlings were transplanted into $16 \mathrm{~cm}$ diameter plastic pots with four hills per pot and three plants per hill. All experiments were conducted with rice plants at the tillering stage.

Planthopper used in the experiments were obtained from a stock population maintained at the China National Rice Research Institute (CNRRI; Hangzhou, China). BHP were reared at Yangzhou University in an insect nursery consisting of rice plants covered with cages under natural conditions in cement tanks from April to October. The $\mathrm{BPH}$ were transferred into a greenhouse during winter. Prior to the experiments, the $\mathrm{BPH}$ colony was allowed to reproduce for two generations in an insectary under standard conditions, $26 \pm 2^{\circ} \mathrm{C}$, and photoperiod, 16L:8D, without insecticide application.

\section{dsRNA Synthesis}

We designed gene-specific dsSPF-L primers and amplified a 272bp SPF-L (XM022339255) cDNA fragment using forward and reverse primers containing the $\mathrm{T} 7$ primer sequence at the $5^{\prime}$ ends (Table 1). The amplification program was 35 cycles of $94^{\circ} \mathrm{C}$ for $1 \mathrm{~min}, 94^{\circ} \mathrm{C}$ for $40 \mathrm{~s}$, and $72^{\circ} \mathrm{C}$ for $1 \mathrm{~min}$, with a final extension step of $72^{\circ} \mathrm{C}$ for $10 \mathrm{~min}$. The sequence was verified by sequencing (Invitrogen, Shanghai, China). We used the GFP gene (ACY56286; provided by Chuan-Xi Zhang, Institute of Insect Sciences, Zhejiang University) as control dsRNA and amplified a $688 \mathrm{bp}$ fragment using primers listed in Table 1. For NISPF-L and the control GFP gene, we used the $\mathrm{T}_{7}$ RiboMAX ${ }^{\mathrm{TM}}$ Express RNAi system (Promega, Sunnyvale, CA, United States) for dsRNA synthesis, following the Promega instructions.

We generated sense and antisense dsRNAs in separate $20 \mu \mathrm{L}$ reaction volumes. The dsRNAs were annealed by mixing and incubation at $70^{\circ} \mathrm{C}$ for $10 \mathrm{~min}$, and then cooling to room temperature over $20 \mathrm{~min}$. Two microliters of RNase A solution $(4 \mathrm{mg} / \mathrm{mL})$ and $2 \mu \mathrm{L}$ RNAase-free DNase $(1 \mathrm{U} / \mu \mathrm{L})$ were added to the reaction tube and incubated in a $37^{\circ} \mathrm{C}$ water bath for $30 \mathrm{~min}$. The dsRNA was precipitated by adding $110 \mu \mathrm{L} 95 \%$ ethanol and $4.4 \mu \mathrm{L} 3 \mathrm{M}$ sodium acetate $(\mathrm{pH} 5.2)$, then washed with $0.5 \mathrm{~mL}$ $70 \%$ ethanol and dried at room temperature. The dried product was dissolved in $50 \mu \mathrm{L}$ nuclease-free water. The purified dsRNAs were quantified by spectroscopy. The 3rd instar nymphs were treated with dsRNA orally on an artificial diet amended with dsRNA (Fu et al., 2001), with some modifications to the rearing

TABLE 1 | PCR primers used in this study.

\begin{tabular}{|c|c|c|}
\hline Primers & Sequence & Product \\
\hline \multicolumn{3}{|c|}{ For dsRNA synthesis } \\
\hline \multirow[t]{2}{*}{ T7-SPF-L-F } & $\underline{\text { TAATACGACTCACTATAGGG }}\left(T_{7}\right)$ & \multirow{2}{*}{$226 \mathrm{bp}$} \\
\hline & AGAGGCCTGCTATCAAACGCTAC & \\
\hline \multirow[t]{2}{*}{ T7-SPF-L-R } & $\underline{\text { TAATACGACTCACTATAGGG }}\left(T_{7}\right)$ & \\
\hline & AGATCAACCGAGTCAGTGTTCCA & \\
\hline \multicolumn{3}{|c|}{ For dsRNA synthesis } \\
\hline \multirow[t]{2}{*}{ T7-GFP-F } & $\underline{\text { TAATACGACTCACTATAGGG }}\left(T_{7}\right)$ & \multirow{2}{*}{688 bp } \\
\hline & AAGGGCGAGGAGCTGTTCACCG & \\
\hline \multirow[t]{2}{*}{ T7-GFP-R } & $\underline{\text { TAATACGACTCACTATAGGG }\left(T_{7}\right)}$ & \\
\hline & CAGCAGGACCATGTGATCGCGC & \\
\hline \multicolumn{3}{|c|}{ For real-time PCR } \\
\hline Q-SPF-L-F & AAGAGTGACAGACCTGCCAA & \multirow{2}{*}{$189 \mathrm{bp}$} \\
\hline Q-SPF-L-R & AGGTTCACTGTCCTTGTCACT & \\
\hline$Q-V g-F$ & GTGGCTCGTTCAAGGTTATGG & \multirow{2}{*}{200 bp } \\
\hline$Q-V g-R$ & GCAATCTCTGGGTGCTGTTG & \\
\hline Q-VgR-F & AGGCAGCCACACAGATAACCGC & \multirow{2}{*}{136 bp } \\
\hline$Q-V g R-R$ & AGGCAGCCACACAGATAACCGC & \\
\hline Actin-F & TGGACTTCGAGCAGGAAATGG & \multirow{2}{*}{$186 \mathrm{bp}$} \\
\hline Actin-R & ACGTCGCACTTCAGATCGAG & \\
\hline
\end{tabular}


protocol. Previous results indicated that a $0.05 \mu \mathrm{g} / \mu \mathrm{L}$ dsRNA concentration feeding led to rapid and significant reduction in trehalose phosphate synthase (NITPS) expression level (Chen et al., 2010). We used glass cylinders $(15.0 \times 2.5 \mathrm{~cm}$ diameter $)$ as feeding chambers, with four dsRNA concentrations, 0.0625 , $0.06,0.05$, and $0.03 \mu \mathrm{g} / \mu \mathrm{L}$. The artificial diet $(20 \mu \mathrm{L})$ was held between two layers of stretched Parafilm M membrane enclosed at the two open ends of the chamber (the diet capsule). The diet capsule was replaced every second day. The cylinders were covered with a piece of black cotton cloth and the two ends with artificial diet were exposed to light. Insects fed on the diets by puncturing the inner Parafilm $\mathrm{M}$ membrane of the diet capsule. Experimental insects were transferred into chambers and maintained on artificial diets for 1 day before initiation of the assays. Twenty $3 \mathrm{rd}$ instar individuals were transferred into each chamber, and three chambers were used to create three independent biological replicates. The rearing experiments were carried out in a humidified growth cabinet at $26 \pm 2{ }^{\circ} \mathrm{C}, 80 \% \mathrm{RH}$ and a 16L: $8 \mathrm{D}$ photoperiod; our standard conditions. Mortality was recorded every other day.

\section{Influence of Dietary dsRNA on Biological Performance Parameters}

We determined the effects of dietary dsSPF-L treatments on selected biological performance parameters. We transferred 3rd instar nymphs to capsules containing dsRNA-laced diet. When the nymphs reached fifth (final) instar (10 days), they were collected, individually transferred into a glass jar $(12 \mathrm{~cm}$ high $\times 6 \mathrm{~cm}$ ), and reared on tillering rice plants under standard conditions. One newly emerged, virgin females mated with one newly emerged dsRNA-treated male. One hundred adult females and 200 adult males were collected separately at 2 days after mating for the determination of fresh body weight ( $n=15, N=3$ for mated adult males; $n=15, N=3$ for mated adult females), soluble ovary protein $(n=20, N=3)$ and soluble fat body protein content $(n=20, N=3)$, juvenile hormone (JH) III titer $(n=5$, $N=3$ for mated females or males), ecdysteroid titer $(n=5$, $N=3$ for mated females or males), MAGPs content $(n=20$, $N=3)$, Argine (Arg) content $(n=5, N=3)$ and western blot $(n=30, N=3)$ at 2 days after emergence (DAE). We determined accumulations of mRNAs encoding vitellogenin (Vg; $n=5$, $N=3$ ), and vitellogenin receptor (VgR; $n=5, N=3$ ) at 2 DAE. We dissected 15 mated males and 15 mated females. We prepared ovaries $(n=15)$ for immunofluorescence staining analysis. We also transferred individual newly emerged females or males into glass jars $(12 \mathrm{~cm}$ high $\times 6 \mathrm{~cm})$, and maintained them separately on tillering rice plants under standard conditions. We collected 100 dsGFP-treated virgin males, 100 dsSPF-L-treated virgin males), and 50 virgin females. We measured the fresh body weight ( $n=15, N=3$ for virgins), JH III, and ecdysteroid titers ( $n=5, N=3$ for virgin females) of each insect. Individual pairs were maintained under standard conditions for oviposition. Two sets (one with dsSPF-L-treated males and one with control males) of 15 copulating pairs were set up to record duration of the pre-oviposition period, oviposition period, adult longevity, and fecundity for each pair. Rice stems were replaced daily during the pre-oviposition period, at 2 day intervals during the oviposition period and 3 day intervals during the female longevity period, until the females died. The number of eggs laid on each rice stem were recoded under a light microscope. We recorded fecundity of 15 mated pairs as the average number of eggs laid/pair. We determined NlSPF-L expression patterns in tissues (detailed in the "Results" section) of 20 dsSPF-L treated virgin males $(n=15, N=3)$ at 2 DAE. We used separate virgin males $(n=5, N=3)$ at $1,3,5$, and 7 DAE to determine NlSPF-L silencing efficiency.

\section{Protein Extraction and Determinations}

We prepared soluble proteins from fat bodies and ovaries of 20 mated females using a method similar to Ge et al. (2010b). We isolated individual tissues from adult females at 2 DAE under a zoom streomicroscope (mode XTL20, Beijing Tech Instrument Co., Ltd., Beijing, China) in a cooled petri dish. We isolated tissues into separate, pre-weighed, ice-cold centrifuge tubes and re-weighed the tubes on a Mettler-Toledo electronic balance (EC100 model; 1/10,000 g sensitivity). We added $\mathrm{NaCl}$ solution (0.4 M NaCl: $1 \mathrm{M}$ PMSF; v/v at $20 \mathrm{~mL} \mathrm{NaCl}$ solution/1 $\mathrm{g}$ tissue) into the tubes, homogenized the tissues on ice, and centrifuged the tubes at $12,000 \times g$ at $4^{\circ} \mathrm{C}$ for $20 \mathrm{~min}$. We filtered out the upper lipid layer with glass fibers, then added $\mathrm{ddH}_{2} \mathrm{O}$ ( 1 volume supernatant: $10 \mathrm{ddH}_{2} \mathrm{O}, \mathrm{v} / \mathrm{v}$ ), and centrifuged again at $4,000 \times g$ at $4^{\circ} \mathrm{C}$ for $20 \mathrm{~min}$. We collected the resulting supernatants and held them at $4^{\circ} \mathrm{C}$ overnight. We removed the supernatant and dissolved the protein sediment in $1.5 \mathrm{~mL}$ of prechilled $0.4 \mathrm{M} \mathrm{NaCl}$. We prepared protein samples from male accessory glands (MAGs) of unmated males as reported Ge et al. (2010b) at 2 DAE. We isolated MAGs from 20 males under a zoom-stereomicroscope in a cooled petri dish and placed them in separate, pre-weighted, ice-cold Eppendorf tubes. We added $600 \mu \mathrm{L}$ of a solution (methanol/distilled water/acetic acid/methyl thioethanol; 80:18:2:0.1; v:v:v:v), homogenized the tissues on ice and centrifuged them at $12,000 \times g$ at $4^{\circ} \mathrm{C}$ for $10 \mathrm{~min}$. After removing the lipid layer, we collected the supernatant. We then added $400 \mu \mathrm{L}$ of the solution to the sediment in the tubes, centrifuged again, collected the supernatant and combined both supernatants.

We followed Bradford (1976) to measure protein content using Coomassie Brilliant Blue R250 (Shanghai Chemical Agent Co., Ltd., Shanghai, China). We created a standard curve based on a BSA protein standard (Shanghai Biochemistry Research Institute, Shanghai, China). We determined the absorbance at $595 \mathrm{~nm}$ in a UV755B spectrometer (Shanghai Precision Instrument Co., Ltd., Shanghai, China) and calculated protein contents according to the standard curve. Protein determinations were repeated three times, with three biologically independent samples.

\section{Determination of Arg Content and Hormone Titers}

We followed the instruction from an insect Arg ELISA kit (Qiandu Biological Technology Co., Ltd., Shanghai, China) to measure Arg content in virgin males at 2 DAE. Each treatment and control were repeated three times $(n=5, N=3)$. 
We followed the instructions from an insect double sandwich ELISA kit (Qiandu Biological Technology Co., Ltd., Shanghai, China) for JH III and ecdysteroid titer determinations for virgin males or mated males at 2 DAE. Each treatment and control were replicated three times $(n=5, N=3)$.

\section{Body Weights and Isolation of IROs}

We determined the fresh weights using 15 mated females and, separately, 15 unmated males at 2 DAE. The insects were placed in pre-weighed centrifuge tubes and then weighed using a Mettler-Toledo electronic balance (EC 100 model; 1/10,000 g sensitivity). Each treatment and each control were replicated three times $(n=15, N=3)$.

We isolated IRO ( $n=$ at least 10 males and, separately, females) from four sets of $\mathrm{BPH}$, control males, experimental males, females mated with control males and females mated with experimental males at 2, 4, and $6 \mathrm{DAE}$ in $1 \times$ phosphate buffered saline (PBS: $137 \mathrm{mM} \mathrm{NaCl}, 2.68 \mathrm{mM} \mathrm{KCl}, 1.47 \mathrm{mM} \mathrm{KH}_{2} \mathrm{PO}_{4}$, and $8.10 \mathrm{mM} \mathrm{Na} \mathrm{HPO}_{4}, \mathrm{pH}$ 7.0). We fixed the tissues in $3.8 \%$ formaldehyde in $1 \times \mathrm{PBS}$ for $20 \mathrm{~min}$ at room temperature (Ge et al., 2017b), then washed the IROs with $0.2 \%$ Triton-X-100 (Sigma, United States) in $1 \times$ PBS three times for $10 \mathrm{~min}$. After washing, we photographed the IROs with a Leica DMR connected to a Fuji FinePixS2 Pro digital camera (Germany).

\section{qPCR Analysis}

We extracted total RNA from five unmated control males (virgin males) at 1,3,5, and 7 DAE or untreated mated males and females at 2, 4, at 6 DAE or untreated virgin males and females at 1,3, and 5 DAE, and from virgin males or virgin females and untreated mated female tissues $(n=20, N=3)$ indicated in Results at 2 DAE using a SV Total Isolation System Kit (model Z3100, Promega Corporation, Madison, WI, United States). We synthesized first-stand cDNA in a $10 \mu \mathrm{L}$ reaction volume composed of $0.5 \mu \mathrm{g}$ RNA, $0.5 \mu \mathrm{L}$ PrimeScript RT enzyme mix I, $0.5 \mu \mathrm{L}$ Oligo dT primer $(50 \mu \mathrm{M}), 2 \mu \mathrm{L}$ random hexamers $(100 \mu \mathrm{M})$, $2 \mu \mathrm{L} 5 \times$ PrimeScript Buffer and RNase-free $\mathrm{dH}_{2} \mathrm{O}$, following instructions of the PrimeScript RT Kit (TakaRa Biotechnology, Dalian, China). The cDNA was reverse transcribed at $37^{\circ} \mathrm{C}$ for $15 \mathrm{~min}, 85^{\circ} \mathrm{C}$ for $5 \mathrm{~s}$ and $4^{\circ} \mathrm{C}$ for $5 \mathrm{~min}$. We extracted and reverse transcribed total RNA from all experimental preparations. All qPCR reactions were performed in triplicate with 96-well plates using a CFX96 touch real-time PCR system (Bio-Rad Co., Ltd., Hercules, CA, United States). Each $10 \mu \mathrm{L}$ qPCR reaction mixture consisted of $5 \mu \mathrm{L}$ SYBR mater mix, $0.4 \mu \mathrm{L}$ of each primer $(10 \mu \mathrm{M}), 1 \mu \mathrm{L}$ of $\mathrm{cDNA}$ template equivalent to $50 \mathrm{ng}$ of total RNA and $3.2 \mu \mathrm{L}$ of deionized water. The qPCR program for NlSPF-L was $94^{\circ} \mathrm{C}$ for $2 \mathrm{~min}$, followed by 40 cycles of $94^{\circ} \mathrm{C}$ for $5 \mathrm{~s}, 56^{\circ} \mathrm{C}$ for $30 \mathrm{~s}$, and $72^{\circ} \mathrm{C}$ for $30 \mathrm{~s}$. The program for $\mathrm{NlVg}(\mathrm{AB} 353856)$ and $N l V g R(\mathrm{Gu} 7232977)$ was $94^{\circ} \mathrm{C}$ for $1 \mathrm{~min}$, followed by 35 cycles of $94^{\circ} \mathrm{C}$ for $5 \mathrm{~s}, 60.4^{\circ} \mathrm{C}(\mathrm{NlVg}) / 60.7^{\circ} \mathrm{C}$ $(N l V g R)$ for $30 \mathrm{~s}$, and $72^{\circ} \mathrm{C}$ for $30 \mathrm{~s}$. NlSPF-L (XM022339255), $N l V g$, and $N l V g R$ mRNAs were separately quantified in relation to the stably expressed (Ge et al., 2017a) reference gene, actin1 (EU179846). After amplification, a melting curve analysis was performed in triplicate and the results were averaged. The values were calculated using three independent biological samples and the relative $N I S P F-L$ transcript level was analyzed by $2^{-\triangle \Delta C T}$ method (Livak and Schmittgen, 2001). Each treatment and control were replicated three times $(n=5, N=3)$.

\section{Western Blot Analysis}

We conducted SDS-PAGE and immunoblotting as previously described (Ge et al., 2019). In brief, the fat bodies (for $\mathrm{NlVg}$ isolation) of treated or control females $(n=20)$ were homogenized using a Tissue Protein Extraction Kit (CWBIO, Taizhou, China). After three freeze/thaw cycles, we centrifuged the homogenates at $12,000 \times g, 4^{\circ} \mathrm{C}$ for $30 \mathrm{~min}$ and determined protein content in the supernatants following the BCA method (CWBIO, Taizhou, China). We mixed the equivalent protein (30 $\mu \mathrm{g}$ ) with $6 \times$ loading buffer for SDS-PAGE (Beyotime, Shanghai, China) and boiled the mixture for $10 \mathrm{~min}$. Following centrifugation at $12,000 \times g$ for $10 \mathrm{~min}$, we cooled the samples to room temperature and then loaded them on $8 \%$ or $12 \%$ SDSPAGE gels. After separating the proteins, the gels were electroblotted onto nitrocellulose (NC) membranes $(0.45 \mu \mathrm{m}$, Solarbio, Beijing, China) using a criterion wet transfer blot apparatus (170471, Bio-Rad, Hercules, CA, United States) at constant $300 \mathrm{~mA}$ for $3 \mathrm{~h}$ and then washed them with Tris-buffered saline (TBS) for $5 \mathrm{~min}$ at room temperature. Blots were blocked at room temperature for $1 \mathrm{~h}$ in TBS containing 0.1\% Tween 20 (TBST) and $5 \%(\mathrm{w} / \mathrm{v})$ non-fat powdered milk, followed by washing three times for $5 \mathrm{~min}$ each with TBST. Blots were incubated overnight at $4^{\circ} \mathrm{C}$ with the primary antibody diluted in TBST (1:2000). Blots were then washed three times in TBST for 10 min each and probed with HRP-conjugated goat anti-rabbit IgG (CWBIO, Taizhou, China) as the second antibody in TBST (1:3000). Following extensive rinsing, the immunoreactivity was visualized using ECL reagent (Bio-Rad, Hercules, CA, United States) in the Molecular Imager ChemiDoc XRS System (Bio-Rad). Quantitative analysis of the visible bands in western blot was performed using the ImageJ program ${ }^{1}$ (Schneider et al., 2012). The procedure entails four steps: (a) converting the analyzed file to Grayscale format and subtracting the background with a value of 50; (b) converting the analyzed file to bright band and calculating the density information of a band by hand-drawing an area corresponding to its borders. The information contains area size, mean gray value, minimum and maximum gray value, and integrated density (IntDen); (c) extracting the IntDen values of all the measured bands; $(\mathrm{d})$ normalization by dividing the Vg IntDen values against the $\beta$-actin values. We obtained the primary antibody, anti- $\beta$-actin, from Abcam (Cambridge, United Kingdom), and anti-rabbit NlVg was generously gifted by Prof. Haijun Xu (Zhejiang University).

\section{Immunofluorescence Microscopy}

We adapted the protocol of Zhuo et al. (2018) for NlVg antibody staining. We isolated ovarioles from ovaries of mated females at 2 DAE in cold PBS ( $\mathrm{pH}$ 7.4). After washing three times in PBS, we fixed the tissues in $4 \%$ paraformaldehyde for $2 \mathrm{~h}$ at room temperature and washed them in PBST containing $0.1 \%$ Triton X-100 three times for $30 \mathrm{~min}$ each. We blocked

\footnotetext{
${ }^{1}$ https://imagej.nih.gov/ij/
} 
the specimens in PBST containing 5\% normal goat serum for $4 \mathrm{~h}$ at room temperature, followed by incubation with antiNlVg (1:500, gifted by Prof. Haijun Xu, Zhejiang University) in PBST containing 2\% normal goat serum and 3\% BSA overnight at $4^{\circ} \mathrm{C}$. After rinsing with $\mathrm{PBS} 3 \mathrm{X}$, for $5 \mathrm{~min}$ each, under dim light, we added Alexa Fluor 488-labeled goat anti-rabbit secondary antibody (1:500) (Beyotime, Shanghai, China) in PBST containing 2\% goat serum and 3\% BSA. We incubated the samples at room temperature for $1 \mathrm{~h}$ and counterstained the nuclei with $100 \mathrm{nM}$ 4,6-diamidino-2-phenylindole (DAPI) (Beyotime, Shanghai, China) in PBST for $10 \mathrm{~min}$ at room temperature. We mounted the specimens on glass slides and washed again with PBS $3 \mathrm{X}, 5 \mathrm{~min}$ each and viewed them under a Leica TCS SP5 confocal microscope (Leica Microsystems, Solms, Germany). We observed DAPI (blue) and NlVg (green) fluorescence at 405 and $488 \mathrm{~nm}$, respectively, with gain and offset set to $1 \%$ and the high voltage set to $400-600$. We used a line sequential scanning mode for image capture at a $1,024 \times 1,024$ pixel resolution.

\section{Phylogenetic Analyses}

We constructed a phylogenetic tree of SPF-L homologs using the Maximum Likelihood method with MEGA6 software (Tamura et al., 2011; Lu et al., 2016). Phylogenetic analysis of the SPF-L amino sequences comes from BPH and other insects. BPH and other insect SPF-L amino sequences were used as a query to search for homologs in the NCBI mRNA and EST library. All of the homologs were then aligned in a multiple sequences alignment using CLUSTAL X (Jeanmougin et al., 1998) and edited with GeneDoc software.

\section{Population Growth}

We carried out a population growth experiment following Ge et al. (2017b), using two treatment groups: females mated with control males and females mated with experimental males. We arranged the experiment in a randomized complete block design with five replicates. Four pairs of newly emerged BPHs were released onto rice plants at the tillering stage and covered with a nylon cylindrical cage $(20 \mathrm{~cm}$ diameter $\times 80 \mathrm{~cm}$ height, screen size: 80 -mesh) in each pot. When neonates of the next generation emerged, we checked each treatment group daily and counted the neonates. We transferred them into new plastic plots as just described until the original females died. We examined the neonates every 2 days until adult emergence and recorded numbers of females and males. We counted all adults from the next generation, recorded the numbers of unhatched eggs on the rice sheaths on which the adults had been feeding and recorded hatch rates as all adults/all adults plus unhatched eggs. A population growth index (PGI) is expressed by the ratio of $N 1 / N 0$, where $N 1$ is the total number of adults from the next generation divided by $\mathrm{N} 0$, the number of adults released $(\mathrm{NO}=8)$.

\section{Statistics Analyses}

Normality and homogeneity data of variance was examined by the Shapiro-Wilk test and Bartlett test, which showed that no transformations were needed. RNAi silencing efficiency of $S P F-L$ at different DAE (Figures 1B, 4H) was performed with using Student's t-test. A one-way ANOVA followed by Tukey's honestly significant difference (HSD) multiple comparison test was performed to analyze other data. All analyses were performed with the data procession system (DPS) of Tang and Feng (2002). Significant differences were considered at $P<0.05$. All values are expressed as mean \pm SEM. The relative gray values for western blot analysis were determined in the NIH ImageJ software (see text footnote 1).

\section{RESULTS}

\section{Dietary dsSPF-L Treatments Influenced Mortality}

From the 3rd instar to 5th instar nymph, mortality of nymph $\mathrm{BPH}$ increased with increasing dietary dsSPF-L concentrations compared to dsGFP treatment control (Figure 1A; $F=186.3$, $\mathrm{df}=4,14, P<0.001)$. Mortality of nymph $\mathrm{BPH}$ feeding 0.05 and $0.03 \mu \mathrm{g} / \mu \mathrm{L}$ dsSPF-L concentrations were not significantly difference (Figure 1A). The silencing efficiencies of high three dietary dsRNA concentrations were similar at approximately $70 \%$ compared to dsGFP treatments controls (Figure 1B; $F=105.4$, $\mathrm{df}=3,23, P<0.001)$ and the lowest dose was not different from controls $(F=2.7, \mathrm{df}=1,8, P=0.1769)$. We selected the $0.05 \mu \mathrm{g} / \mu \mathrm{L}$ dsRNA concentration for all experiments based on mortality and silencing efficiency of $N I S P F-L$ in virgin $\mathrm{BPH}$ males at $2 \mathrm{DAE}$.

\section{Phylogenetic and Sequence Analyses of the BPH SPF-L}

We opted to do these amino acid sequences since online blast results showed the homology of them was all beyond $60 \%$ against the target one. Thus, some sequences with less similarity are not displayed. Phylogenetic analysis based on amino acid sequences indicates that selenoproteins from the species listed in Figure 2 clustered reasonably close together. In particular, the four hemipteran proteins were quite close, like Myzus persicae, Rhopalosiphum maidis, and Cimex lectularius, suggesting the SPF-Ls were evolutionarily conserved. Multiple sequences alignment of $B$. mori, $H$. armigera, A. mellifera, $N$. lugnes showed conserved residues primarily from Sepl15/SelM redox domain as a thiroredoxin-like and active-site redox motif, and have selenocystine-containing CXXC motifs and CXXC derived motifs as active-residues (Figure 3).

\section{NISPF-L Expression Characteristic and Silencing Efficiency Analysis}

We recorded relative accumulations of mRNA encoding SPF-L. Thoraces produced the lowest amounts, fat bodies slightly more and IRO much higher levels, up approximately 7.9-fold compared to heads in virgin male at $2 \mathrm{DAE}$ (Figure $4 \mathrm{~A} ; F=2,319.9, \mathrm{df}=3$, $11, P<0.001)$. SPF- $L$ mRNA expression level in virgin males was no significant difference at 1, 3, and 5 DAE (Figure 4B; $F=4.3$, df $=2,8, P=0.0684)$, and in mated males at 4 and 6 DAE was higher than that of mated males at $2 \mathrm{DAE}$ (Figure 4C; $F=53.2$, $\mathrm{df}=2,8, P=0.0002$ ). Meanwhile, 


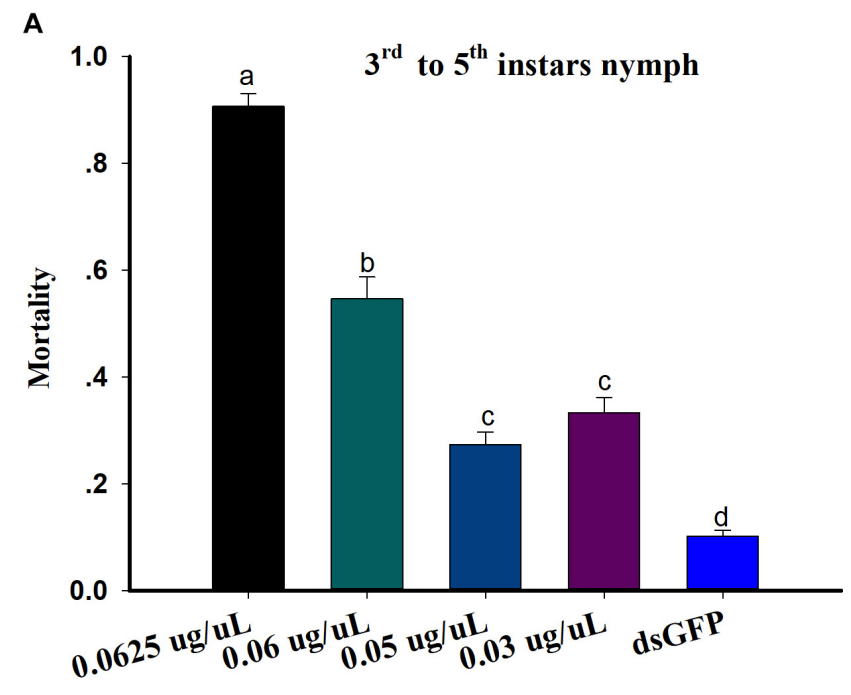

dsRNA concentrations

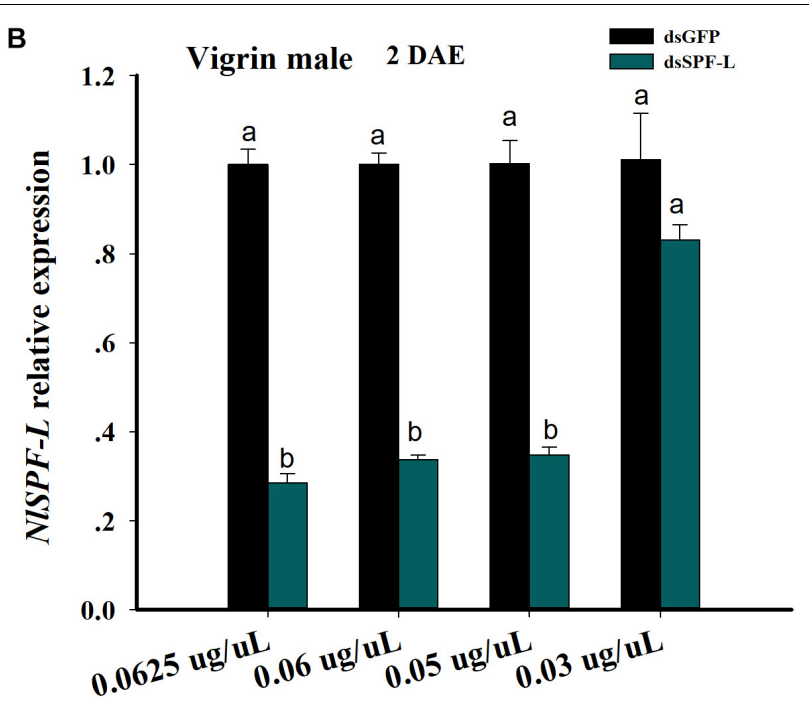

dsRNA concentrations

FIGURE 1 | dsRNA treatments influence nymphal BPH mortality and accumulation of mRNAs encoding NISPF-L. (A) The histogram bars show mean BPH male and nymph mortality as proportions. The error bars indicate one SEM, $n=50, N=3$ independent biological replicates. (B) The histogram bars show mean silencing efficiency of NISPF- $L$ in dsRNA-treated males at 2 DAE, $n=5, N=3$ independent biological replicates (Student's $t$-test). The histogram bars with different letters are significant difference at $p<0.05$.

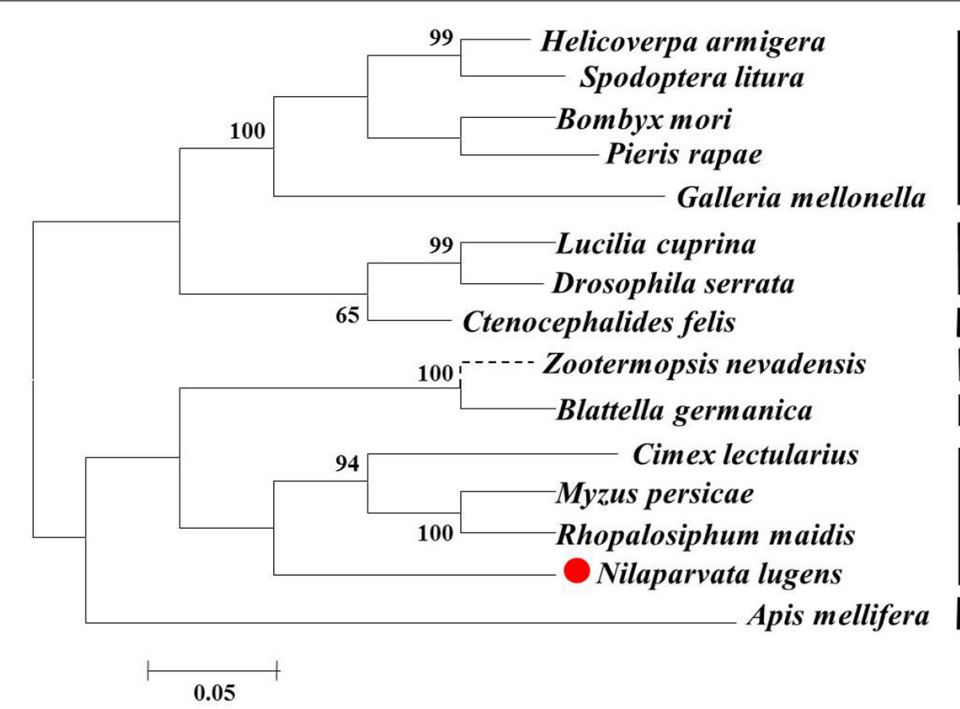

LEPIDOPTERA

DIPTERA

SIPHONAPTERA

ISOPTERA

BLATTODEA

HEMIPTERA

HYMENOPERA

\section{ARTHROPODA}

FIGURE 2 | Phylogenetic analysis, based on amino acid sequences, of the SPF-L sequences from BPH and other insects. Sequences were retrieved from GenBank protein database, including Helicoverpa armigera (XP021184618), Zootermopsis nevadensis (XP021941207), C. lectularius (XP014242913), Blattella germanica (PSN58039), Bombyx mori (XP004927858), Spodoptera litura (XP022829415), Pieris rapae (XP022120009), Myzus persicae (XP022167433), Rhopalosiphum maidis (XP026813571), Apis mellifera (XP394140), Lucilia cuprina (XP023302715), Ctenocephalides felis (XP026461767), Drosophila serrata (XP020809590), and Nilaparvata lugens (XP022194947). The phylogentic tree of SPF-L homologs was constructed using the Maximum Likelihood method with MEGA6 software. Bootstrap values are shown in the nodes. Branch lengths are proportional to sequence divergence. The scale bar indicates the average number of amino acid substitutions per site.

we found $S P F-L$ expression level in fat body of virgin females was higher than that of other tissues at 2 DAE (Figure 4D; $F=9.6$, df $=3,11, P=0.0049$ ), and no significant difference in virgin females at 1,3 , and 5 DAE (Figure 4E; $F=0.29$, $\mathrm{df}=2,8, P=0.7594)$, SPF-L expression level of fat body and
IRO in mated females at 2 DAE was aslo higher than those of head and thorax in mated females (Figure 4F; $F=24.1$, $\mathrm{df}=3,11, P=0.0002)$, in mated females at 4 and 6 DAE was higher than that of mated females at 2 DAE (Figure 4G; $F=547.6, \mathrm{df}=2,8, P<0.001)$. 


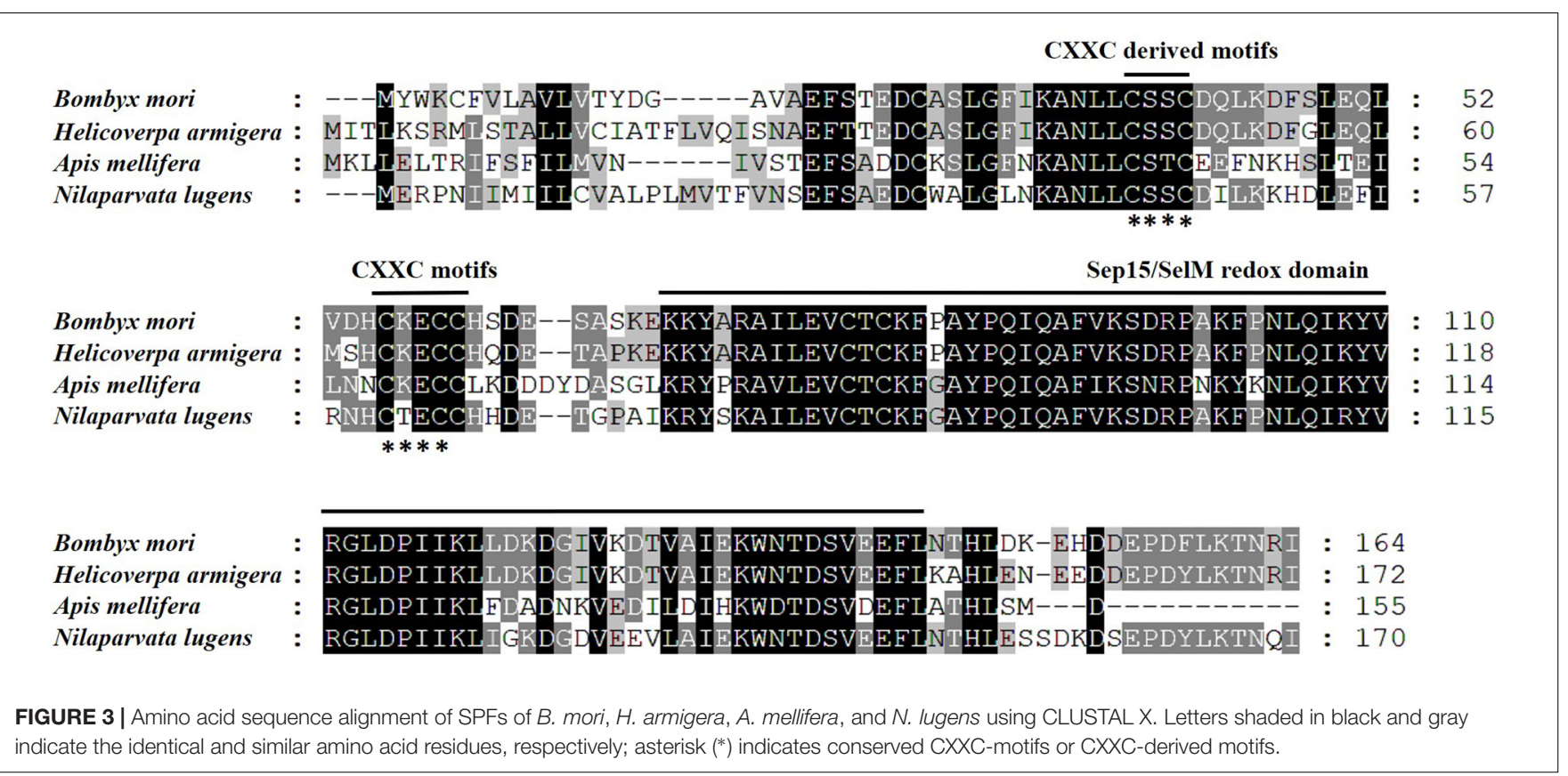

These results indicated $S P F-L$ mRNA specifically expressed in fat body and IRO of virgin males, and high expression in fat body of virgin females. SPF- $L$ mRNA expression levels was relatively stable in either the virgin females or the virgin males, $S P F-L$ expression level was markedly increased in either mated females or mated males, suggesting SPF-L transferred to female via copulation. dsSPF-L treatments led to reduced $(F=500.1$, $\mathrm{df}=1,23, P<0.001)$ accumulations of mRNAs encoding SPF-L compared to dsGFP treatments at 1, 3, 5, and 7 DAE (Figure $4 \mathbf{H}$ ), although the amounts of reduction were similar at all 4 days $(F=2.1, \mathrm{df}=3,23, P=0.1367)$. Also, no significant interactions between RNAi and DAE were observed $(F=2.2$, df $=3,23$, $P=0.1328$ ) (Figure 4H).

\section{Influence of Silencing SPF-L on Males}

In virgin males, dietary dsSPF-L treatments led to reduced MAGP content $(F=421.2, \mathrm{df}=1,5, P=0.0001)$, down by $39 \%$ compared to dsGFP treatments (Figure 5A) and to reduced Arg content at $2 \operatorname{DAE}(F=32.4, \mathrm{df}=1,5, P=0.0047)$, down by $20 \%$ (Figure 5B). The dsRNA treatments did not influence JH III (Figure 5C; $F=0.6, \mathrm{df}=1,5, P=0.481$ ) or ecdysteroid titers (Figure 5D; $F=1.4$, df $=1,5, P=0.3082$ ), nor body weights (Figure 5G; $F=0.03$, df $=1,5, P=0.8703)$. In mated males, the dsRNA treatments led to increased JH III titers (Figure 5E; $F=35.2$, $\mathrm{df}=1,5, P=0.004$ ) and decreased ecdysteroid titers (Figure 5F; $F=29.5, \mathrm{df}=1,5, P=0.0056)$ at $2 \mathrm{DAE}$. The treatments did not influence longevity of mated males (Figure 5H; $F=0.046, \mathrm{df}=1$, 29, $P=0.8317)$.

\section{dsSPF-L Treated Males Influence Their Mating Partners}

Mating with experimental males led to reduced fat body $(F=36.4$, $\mathrm{df}=1,5, P=0.0038)$ and ovarian $(F=71.6, \mathrm{df}=1,5$,
$P=0.0011)$ protein contents at $2 \mathrm{DAE}$, as well as to reduced JH III titers (Figure 6C; $F=45.3$, df $=1,5, P=0.0025$ ) in their partners (Figures 6A-C), compared to controls at 2 DAE. Similar experimental matings did not influence ecdysteroid titers (Figure 6D; $F=1.3$, df $=1,5, P=0.3175$ ), body weights (Figure 6E; $F=0.169$, df $=1,5, P=0.7023$ ) at 2 DAE, nor longevity (Figure 6F; $F=0.006$, df $=1,29$, $P=0.9388)$ in partners.

\section{dsSPF-L-Treated Males Influence $\mathbf{N I V g}$ Gene Expression and Vg Synthesis}

Mated with dsSPF-L-treated males led to reduced accumulations of mRNAs encoding NlVg (Figure 7A; $F=46.9$, $\mathrm{df}=1,5$, $P=0.0024$ ), but not NlVgR (Figure $7 \mathrm{~B} ; F=0.285$, $\mathrm{df}=1$, $5, P=0.6218$ ), in their partners, down by $354 \%$ compared to controls at $2 \mathrm{DAE}$. We confirmed the reduced $\mathrm{NlVg}$ expression by western blot analysis (Figure 7C; Gray value $=\mathrm{Vg} / \beta$-actin $=0.77$ ). Fluorescence staining showed that the uptake of $\mathrm{Vg}$ by the follicle cells was impeded in females that mated with dsSPF-L-treated males, but not after mating with control males (Figure 8).

\section{Influence of dsSPF-L-Treated-Males on Female Reproduction}

Mating with dsSPF-L-treated males led to reduced numbers of laid eggs per female $(F=115.5$, df $=1,29, P<0.001)$, down by $56 \%$ compared to controls (Figure 9A); elongated preoviposition periods, up by $20 \%$ (Figure 9B; $F=5.2$, df $=1$, $29, P=0.0299$ ), and no change in the oviposition period (Figure 9C; $F=0.4$, $\mathrm{df}=1,29, P=0.5322$ ). Figure 9D shows total numbers of deposited eggs following experimental matings (1,832 eggs) and control matings $(4,204)$. The influence of experimental matings was significantly lower compared to control matings during two intervals, 1-3 d and 21-24 d. 

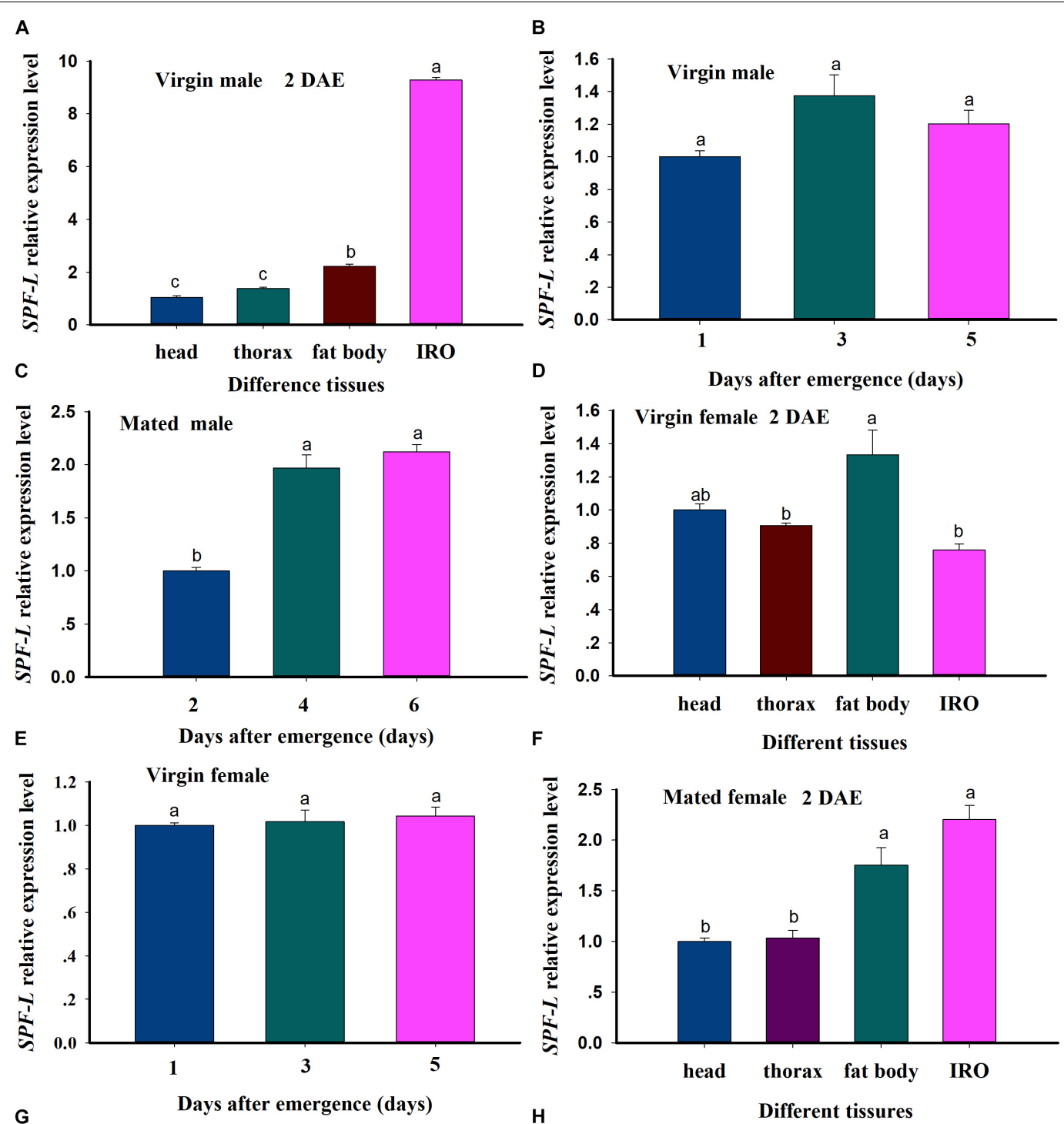

H
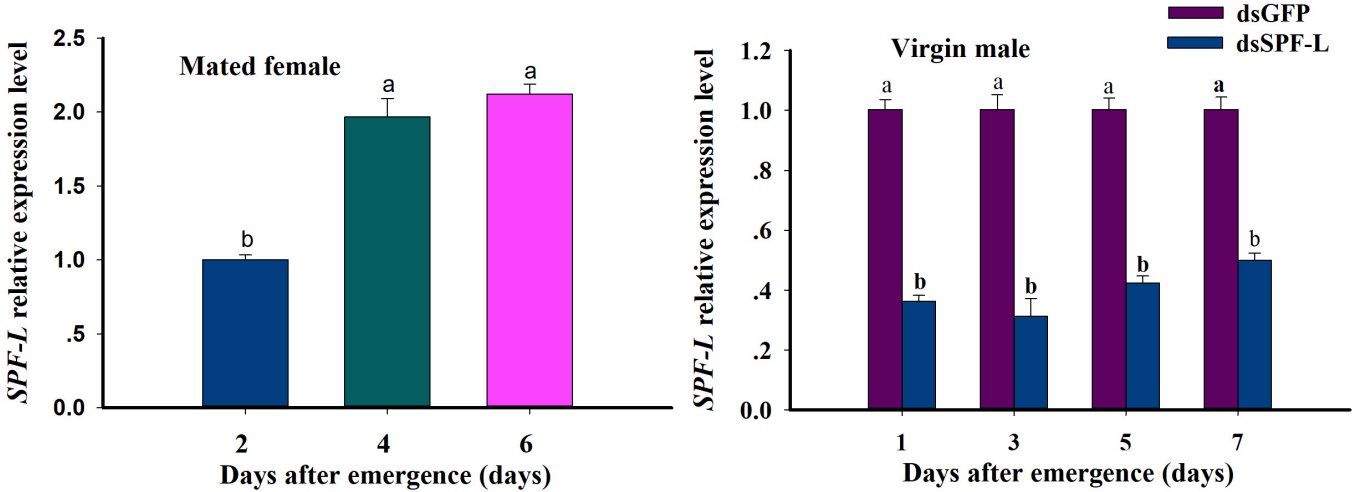

FIGURE 4 | Relative accumulations of mRNA encoding the BPH SPF-L. (A) The histogram bars indicate accumulations of mRNA in the indicated body segments and tissues of virgin male at 2 DAE. (B,C) The histogram bars indicate the mean $S P F-L$ expression level in virgin males and mated males $(n=5, N=3)$. (D,E) The histogram bars indicate the mean SPF- $L$ expression level in the tissues of virgin female $(n=20, N=3)$ and at $2,4,6 \mathrm{DAE}$ in virgin female $(n=5, N=3)$. (F,G) The histogram bars indicate the mean SPF-L expression level in the tissues of mated female $(n=20, N=3)$ and at $2,4,6$ DAE in mated female ( $n=5$, $N=3$ ). The error bars indicate one SEM. The mRNA expression value of adult female head was converted to 1. (H) The histogram bars show mean relative mRNA accumulation ( $n=5, N=3$ independent biological replicates) at 1, 3, 5, and 7 DAE in virgin males and the error bars represent one SEM. Gene expression was normalized to the $\beta$-actin reference gene. The histogram bars with different letters were significant difference at $p<0.05$ (Student's $t$-test). 

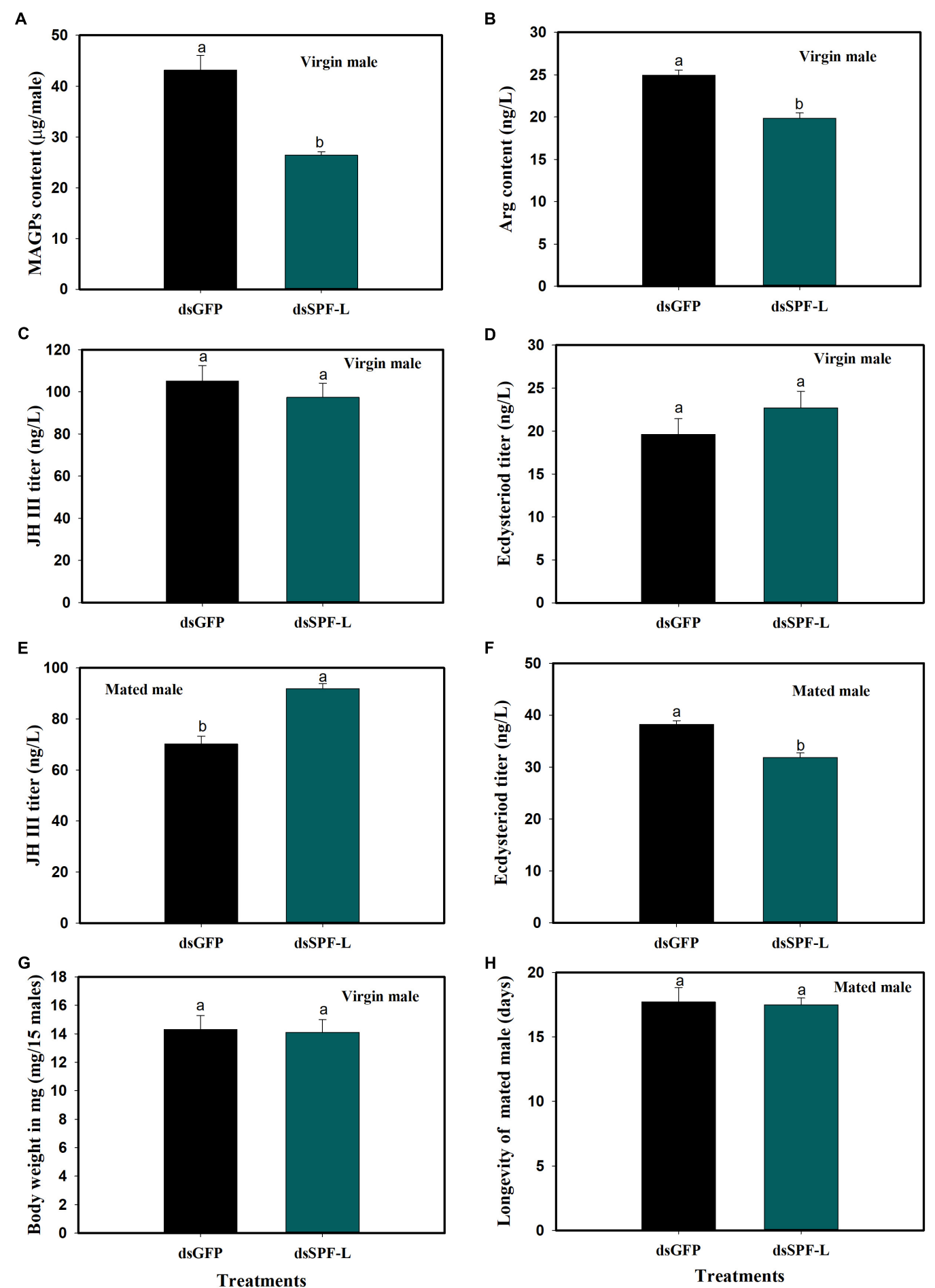

FIGURE 5 | Effects of dsSPF-L treatments on male BPH parameters. (A) The histogram bars show mean of MAGs content in dsSPF-L-treated virgin males at 2 DAE ( $n=20, N=3$ independent biological replicates). (B) The histogram bars show mean of Arg content in dsSPF-L-treatedvirgin males at 2 DAE $(n=5, N=3$ independent biological replicates). (C) The histogram bars show mean JH III titer in dsSPF-L-treated virgin male at 2 DAE ( $n=5, N=3$ independent biological replicates). (D) The histogram bars show mean ecdysteriod titer in dsSPF-L-treated virgin male at 2 DAE ( $n=5, N=3$ independent biological replicates). (E) The histogram bars show mean JH III titer in dsSPF-L-treated mated males at 2 DAE ( $n=5, N=3$ independent biological replicates). (F) The histogram bars show mean of ecdysteroids titer in dsSPF-L-treated mated males at 2 DAE ( $n=3$ independent biological replicates). (G) The histogram bars show mean body weight in dsSPF-L-treated virgin males at 2 DAE ( $n=15, N=3$ independent biological replicates). (H) The histogram bars show mean longevity in dsSPF-L-treated males ( $n=15$ independent biological replicates). All error bars indicate 1 SEM. The histogram bars with different letters were significant difference at $p<0.05$ (Student's t-test). 

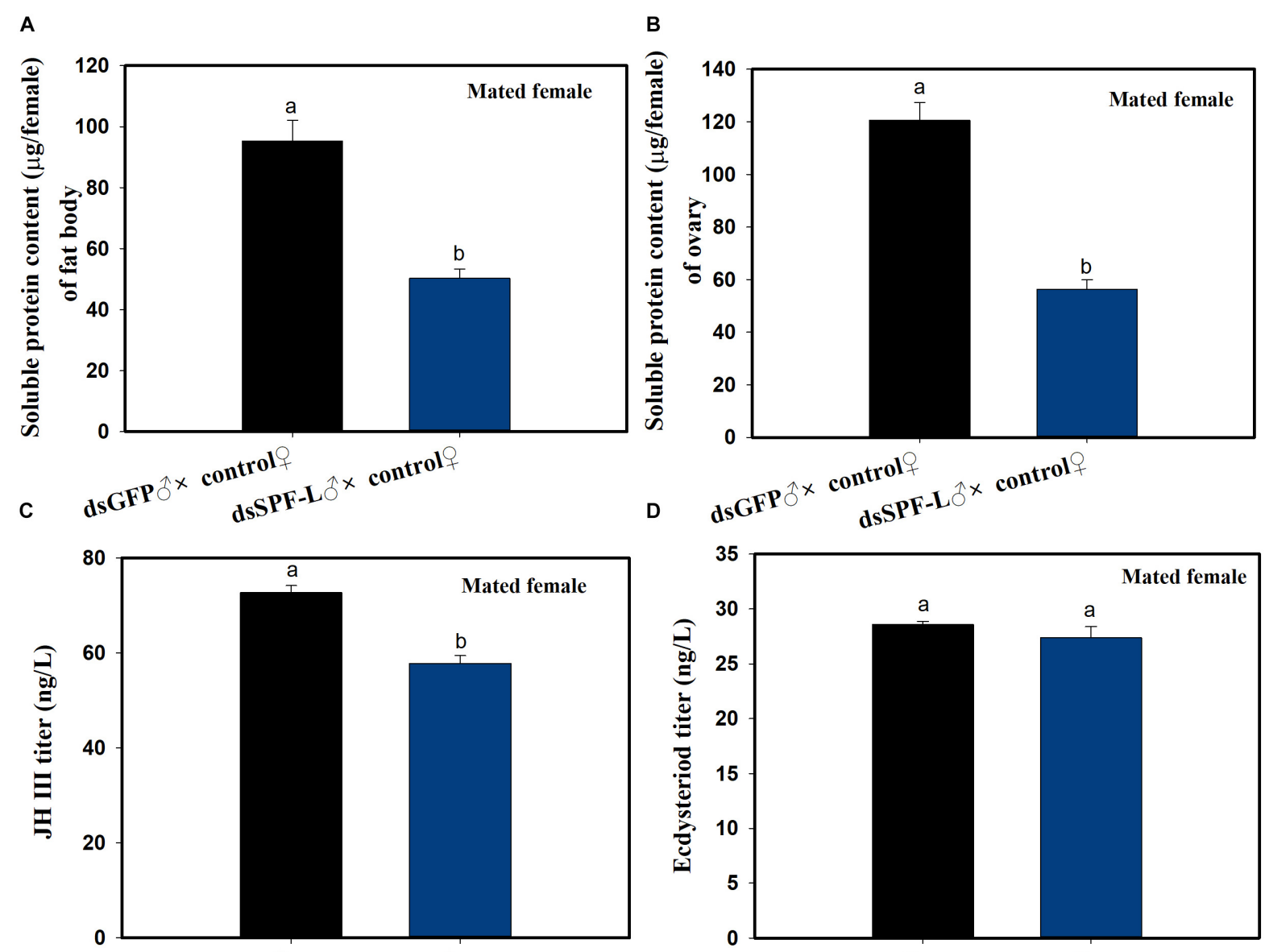

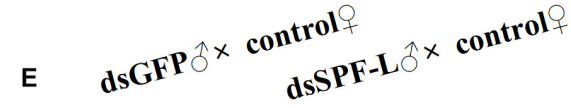

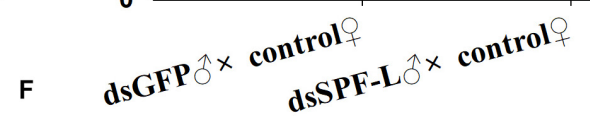

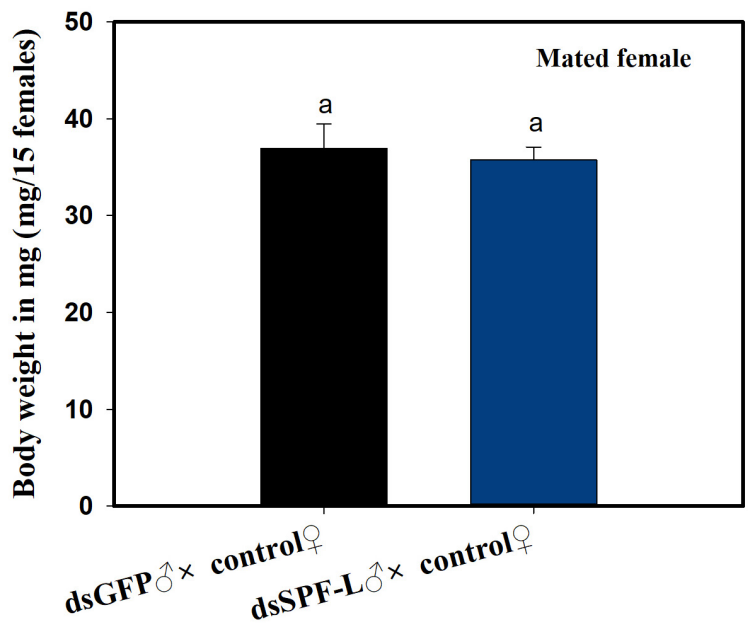

Treatments

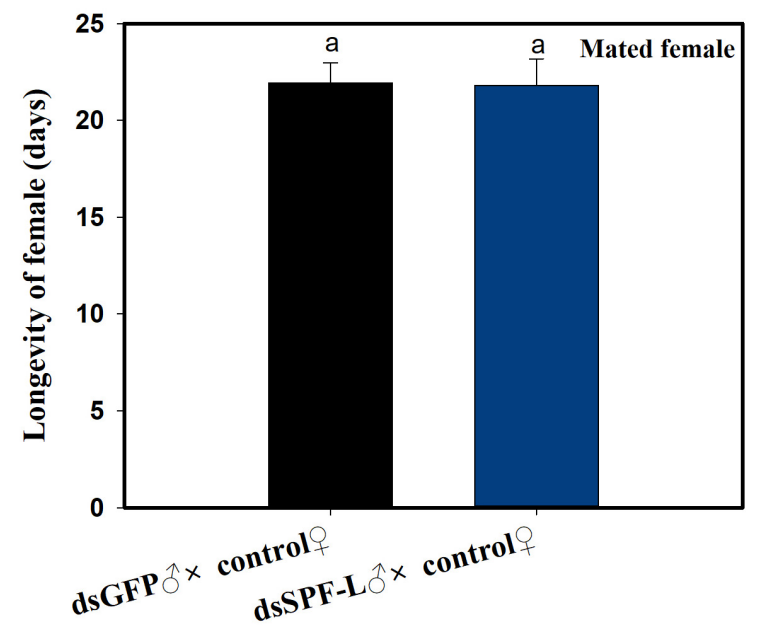

Treatments

FIGURE 6 | The influence of mating with dsSPF-Ltreated males on females at 2 DAE. (A) The histogram bars show mean fat body soluble protein content in mated females ( $n=20, N=3$ independent biological replicates). (B) The histogram bars show mean ovarian soluble protein content in mated females $(n=20, N=3$ independent biological replicates). (C) The histogram bars show mean JH III titer ( $n=3$ independent biological replicates). (D) The histogram bars show mean ecdysteriod titer ( $n=3$ independent biological replicated). (E) The histogram bars show mean body weight $(n=15, N=3$ independent biological replicated). (F) The histogram bars show mean longevity of females ( $n=15$ independent biological replicated). All error bars indicate 1 SEM. The histogram bars with different letters were significant difference at $p<0.05$ (Student's $t$-test). 


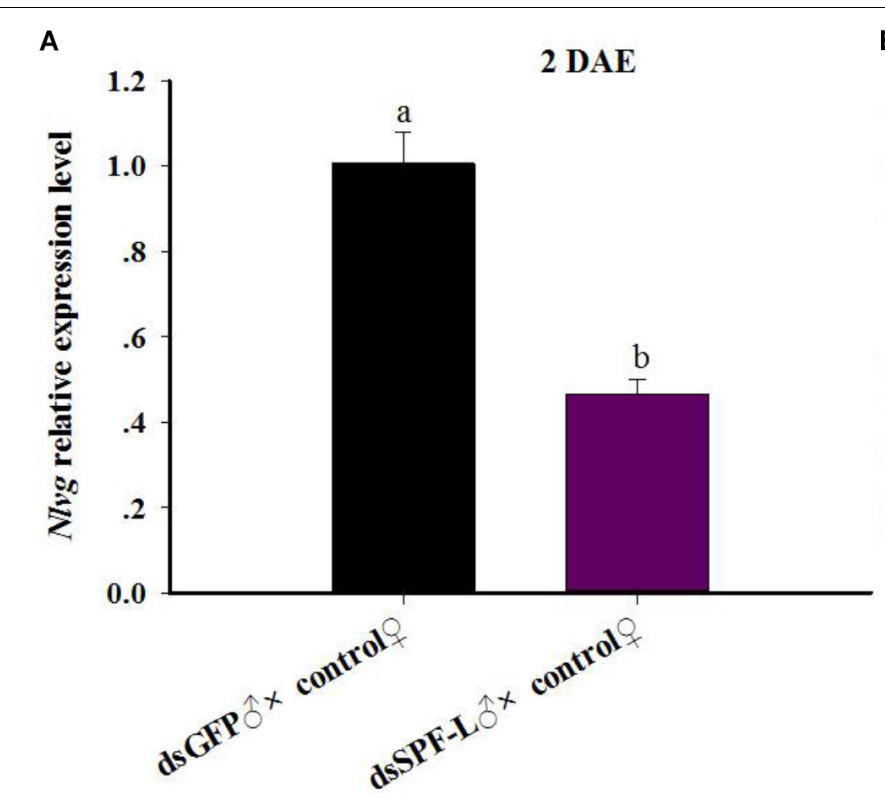

Treatments

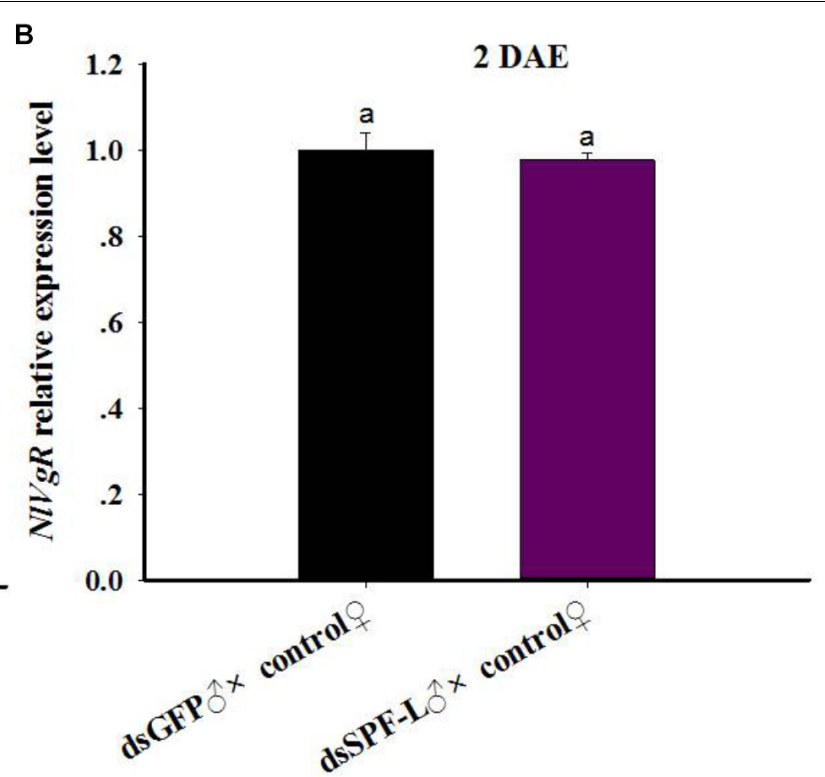

Treatments

2 DAE

C

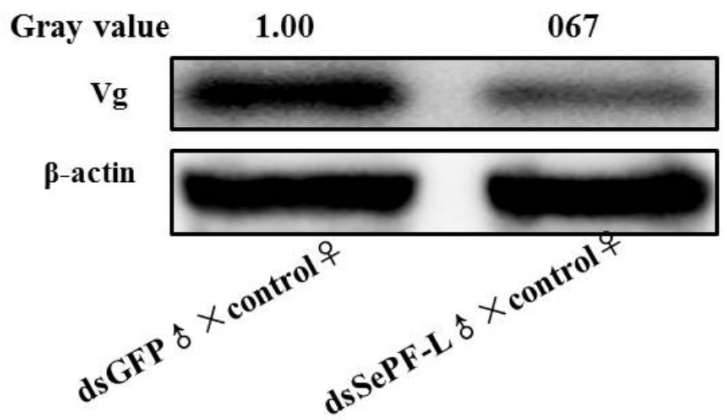

FIGURE 7| The influence of mating with dsSPF-Ltreatedmales on accumulations of mRNAs encoding Vg, VgR, and Vg protein synthesis. (A) The histogram bars show mean NIVg expression level at 2 DAE $(n=5, N=3)$. (B) The histogram bars show mean NIVgR expression level at 2 DAE $(n=5, N=3)$. (C) Western blot analysis of mating with dsSPF-L-treated males led to reduced fat body Vg protein levels of $V g$ using antibody-rabbit $V g$ in at $2 \mathrm{DAE}(n=5, N=3$ ). $\beta$-actin antibody was used as the loading control. The relative gray values normalized to $\beta$-actin were marked above corresponding bands. The relative gray values of dsGFP treatment was looked on as 1. The histogram bars with different letters were significant difference at $p<0.05$ (Student's $t$-test).

For the 24-27 d interval, control matings led to very few deposited eggs and egg laying was abolished after experimental matings (Figure 9D).

\section{dsSPF-L Treatments Led to Disrupted IRO Development in Males and Their Partners}

Sperm are produced in testes, then pass out of the testes through ducts called vasa efferentia and collect in seminal vesicles. Similar ducts, vasa deferentia, transfer sperm from seminal vesicles into an ejaculatory duct. Males also feature accessory glands in which seminal fluids are produced. Some MAGs also produce spermatophores, in which sperm and seminal fluids are transferred to mating partners (Figure 10F).
We found that dsSPF-L treatments led to disrupted IRO development in males, seen as transparent testicular tubules and vas efferent, while vas deferens became thin and lightcolored (Figures 10A-C) compared to controls at 2, 4, and 6 DAE (Figures 10D-E). After mating with dsSPF-L-treated males, the ovarioles contained far fewer ripe banana-shaped oocytes (Figures 10G-I) compared to controls (Figures 10J-L) at 2, 4, and 6 DAE. We recorded retarded ovarian development with fewer mature eggs in mating partners of dsSPF-L-treated males (Figures 10G-I) over the same time-frame. These females absorbed less Vg into follicle cells which impeded oocytes and ovarian development (Figures 10G-I).

Matings with dsSPF-L-treated males led to very small spermathecae compared to spermathecae from controls (Figures 11A-B). This may be due to the absence of transferred 


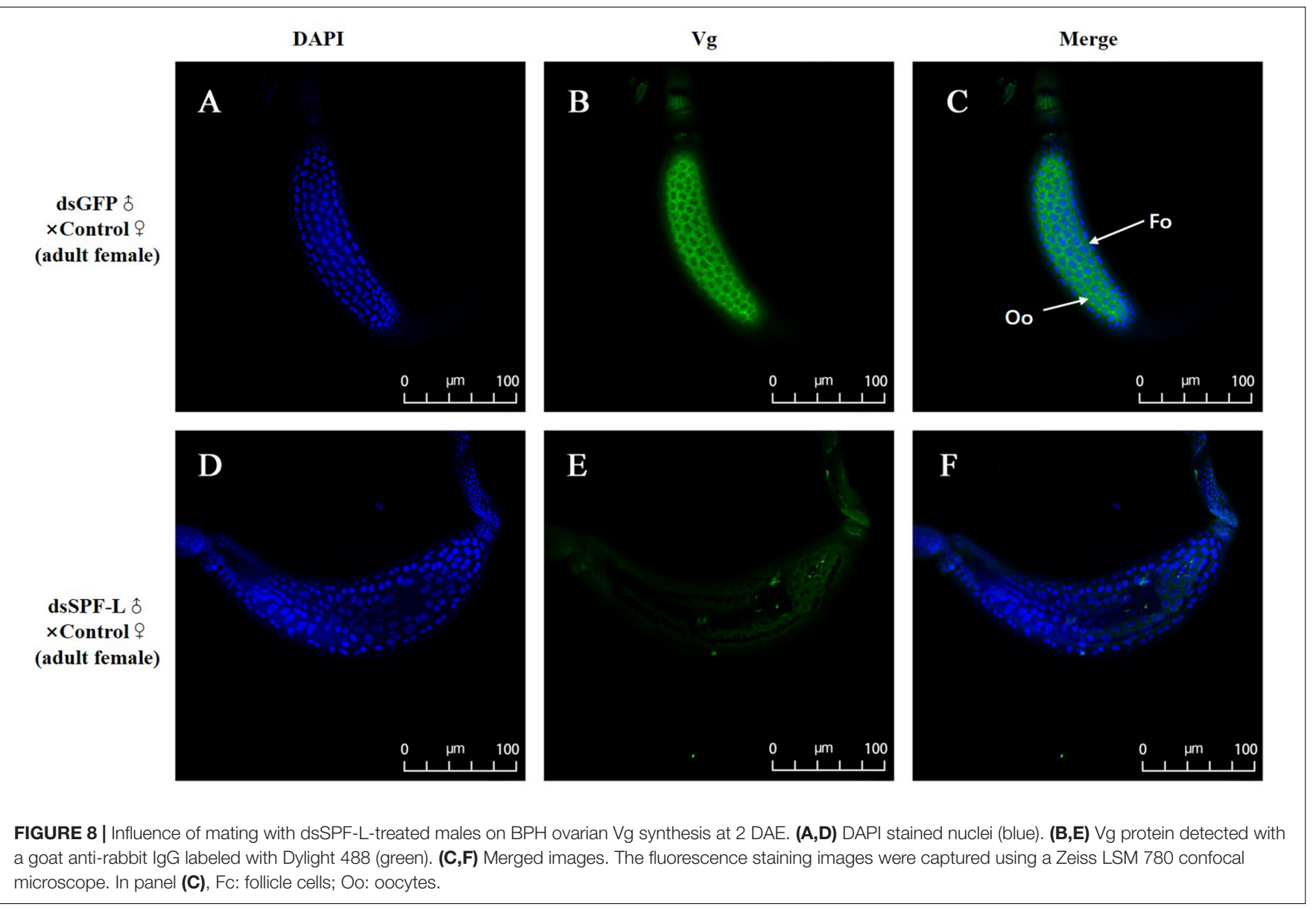

sperm because the vas deferens is very much smaller in experimental, compared to control, males (Figures 11C-D). The smaller vas deferens may not support sperm transfer. Figures 11E-F shows the anatomical locations of the spermaducts shown in Figures 11C-D.

\section{Matings With dsSPF-L-Treated Males Led to Reduced Offspring, Hatching Rate, Gender Ratio, and Population Index}

Matings with dsSPF-L-treated males led to reduced numbers of offspring (Table 2; $F=78.7$, df $=1,9, P<0.001$ ), down by $43 \%$ compared to controls, hatch rates $(F=81.2, \mathrm{df}=1,9$, $P<0.001$ ), down by $28 \%$ and PGI, without influencing the gender ratio of offspring.

\section{DISCUSSION}

The data presented in this paper form lines of evidence supporting our hypothesis that male SPF-L influences female fitness, seen as increased offspring. Several points make up the argument. First, phylogenetic analysis shows the BPH SPF-L clusters most closely with two other hemipteran SPF-Ls at the amino acid level. Second, the gene is expressed in several tissues, most highly in the IRO of virgin males. Third, silencing the gene with a dietary dsRNA construct led to reduced MAGP and reduced Arg in unmated males and influenced JH III and ecdysteroid titers in mated males. Fourth, silencing NlSPF-L in males led to reduced fat body and ovarian protein contents and reduced JH III titers in their mating partners. Fifth, mating with dsSPF-L-treated males led to substantial reductions in Nlvg expression and reductions in Vg protein in females. Sixth, mating with dsSPF-L-treated males led to reduced female reproductive fitness, registered as reduced oviposition, reduced offspring numbers, reduced egg hatch rates and reduced PGI values. Finally, dsSPF-L treatments led to serious disruptions in IRO development in males and in their mating partners. We infer our hypothesis is potently supported.

SPF acts in oxidative stress, endoplasmic reticulum stress and carcinogenesis (Ferguson et al., 2006). It is conserved across animal phyla, and we understand that genes in Caenorhabditis elegans, Brugia malayi, and Drosophila melanogaster encode homologous proteins that contain cysteine in place of selenocysteine (Gladyshev et al., 1998). The dendrogram protein highlights a consecutive 75 amino acid segment (75-149) homologous to other hemipteran species, $M$. persicae and $R$. maidis. We infer that SPFs are relatively conserved in evolutionary processes. The protein 

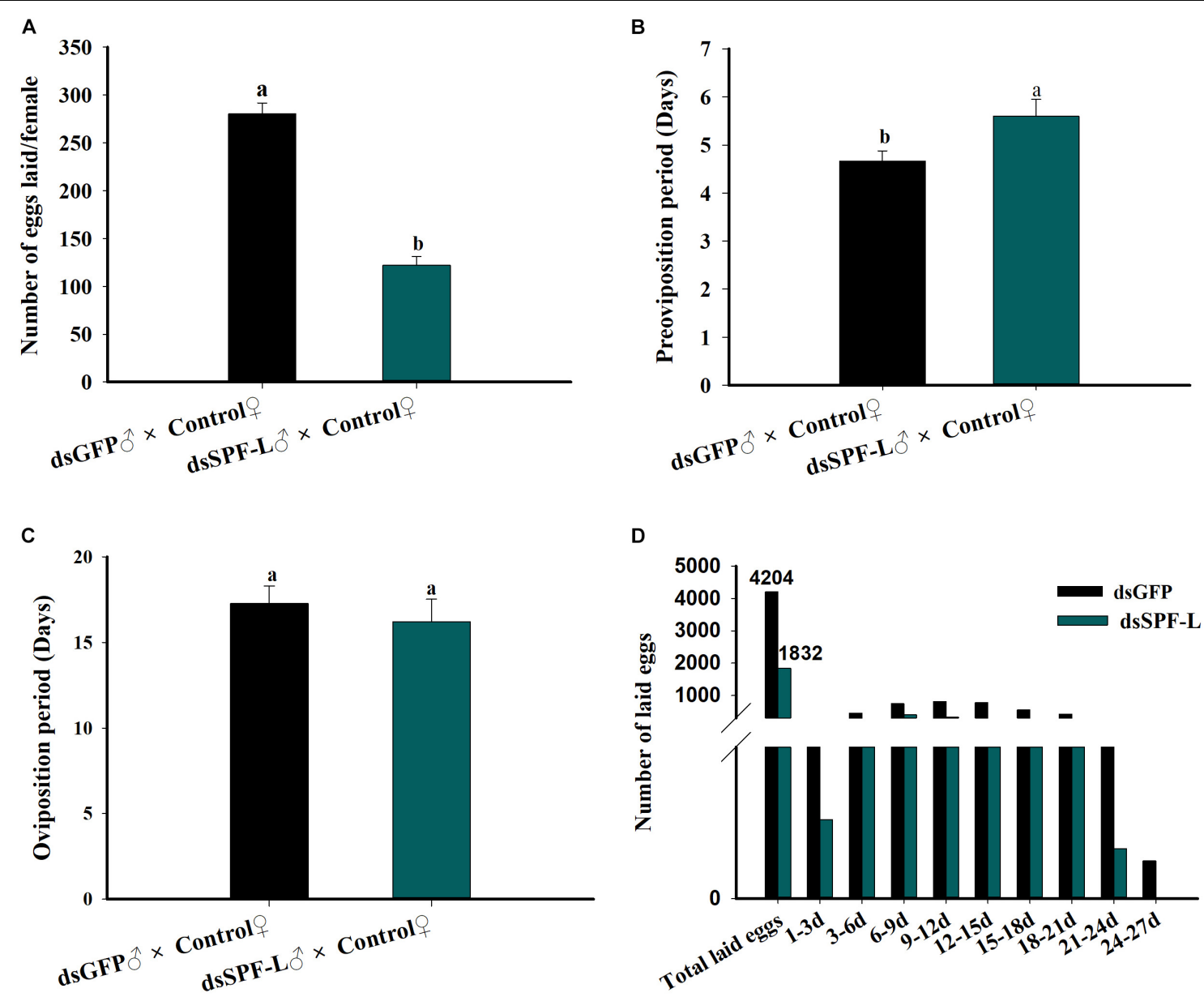

\section{Treatments}

FIGURE 9 | Influence of mating with dsSPF-L treated males on female reproductive parameters. (A) The histogram bars show mean numbers of eggs deposited ( $n=15$ independent biological replicates). (B) The histogram bars show duration of the pre-oviposition period (days) ( $n=15$ independent biological replicates). (C) The histogram bars show the duration of the oviposition period (days) ( $n=15$ independent biological replicates). (D) The histogram bars show numbers of eggs deposited during the indicated oviposition intervals in days. $n=15$ mating pairs. The histogram bars with different letters are significant difference at $p<0.05$ (Student's t-test)

sequence alignment of the BPH SPF-L also shows conserved residues primarily from Sep15/Se1M redox domain (amino acids 75-149) as a thioredoxin-like and active-site redox motif, harboring selenocysteine-containing CXXC-like motifs (amino acids 61-64) and CXXC-derived motifs (amino acids 43-46) as active-site cysteine residues. We infer from these amino acid features that the BPH SPF-L also acts in oxidative stress.

Insect seminal fluid proteins are important for fertilization and for males in sexual competition, such as manipulating post-mating physiology and behavior in females (LaFlamme and Wolfner, 2013). The current results indicated that SPF-L mRNA was expressed in males, unmated females, and mated females. SPF-L expression level significantly increased in mated males and female, suggesting SPF-L mRNA level was induced by copulation. Yu et al. (2016) reported that SPF-L was found in the male reproductive tract and the female reproductive tract, and was transferred to females after mating. Selenoproteins in human seminal fluid are likely important for protecting during storage (Michaelis et al., 2014). Silencing the BPH SPF$L$ in males led to reduced MAGPs contents. MAGPs help ensure male paternity via several mechanisms, such as sperm mobility, sperm storage, stimulation of ovulation/oviposition, and egg protection (Gillott, 2003). Two examples of this are the example of the, cricket, Teleogryllus commodus, mating system, males transfer compounds that release egg-laying behavior in newly mated females (Loher et al., 1981). This promotes egg fertilization before female partners can re-mate with other males. Similarly, Garcia-Gonzalez and Simmons (2005) reported that sperm viability is positively correlated with paternity assurance in the related cricket, Teleogryllus oceanicus (Leguillou). Thus, seminal fluids contribute to male and, separately, female fitness, here taken as reproductive success and long-term persistence of genes. 


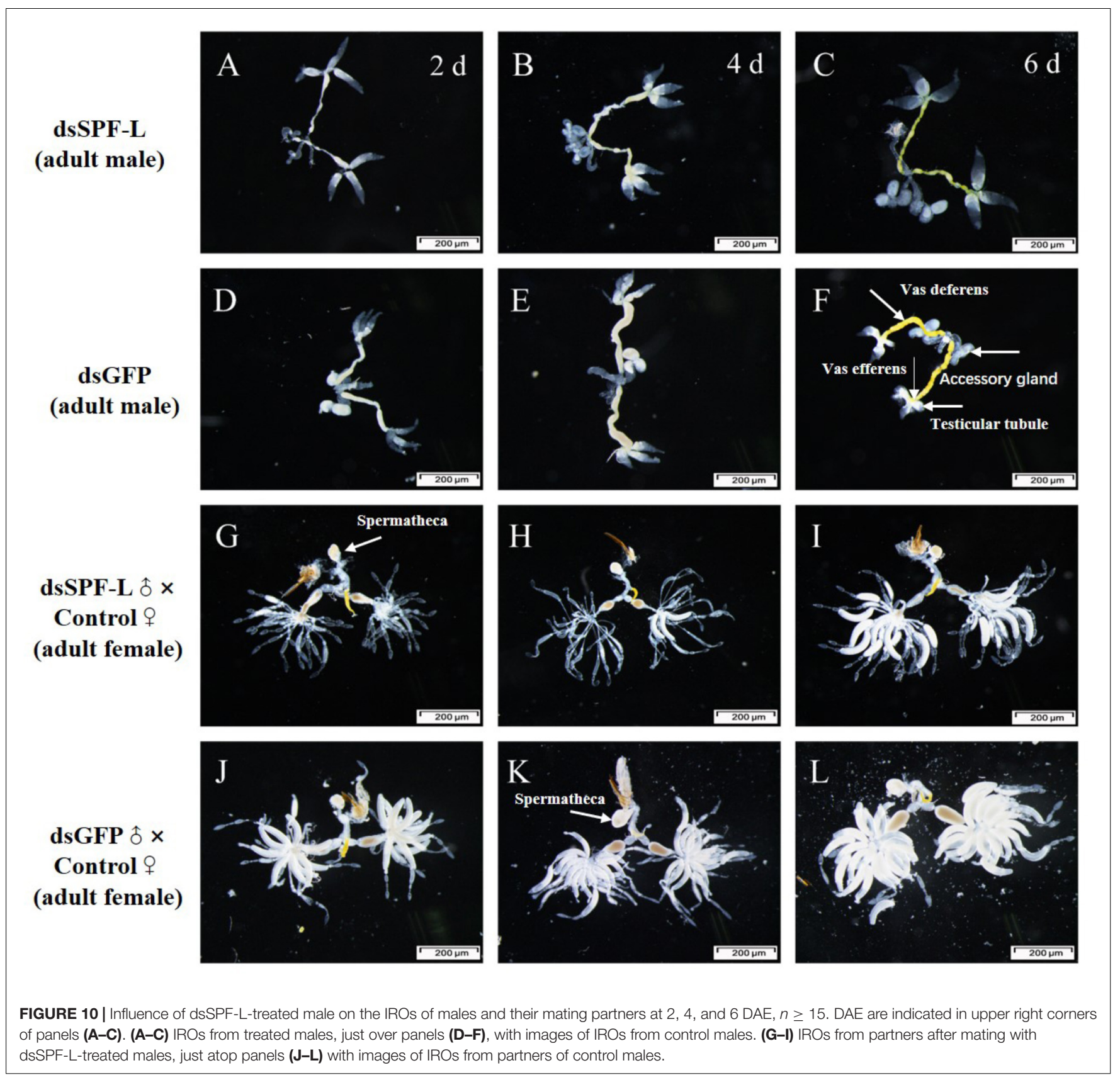

Triazophs exposure enhances BPH reproduction through several mechanisms, one of which is recorded as increased MAGPs transfer to females (Ge et al., 2010a; Wang et al., 2010). NISPF-L silencing also led to reduced transfer of Arg, a nitric oxide (NO) precursor. NO promotes spermatogenesis and enhances fertilization (Zhang and Zheng, 1996; Fujisawa et al., 2001). We infer that dietary dsSPF-L treatments influence spermatogenesis and sperm motility. The images of spermathecal staining indicate reduced sperm transfer from dsSPF-L-treated males. Our interpretation is that the dsSPF-L treatments led to reduced transfer of MAGPs content, Arg content and number of sperm, which translates into reduced egg production and deposition.

Seminal fluids have complex compositions, including multiple inorganic ions, amino acids, carbohydrates, lipids, proteins (sex peptides) and hormones, such as $\mathrm{JH}$ or ecdysteroids (Wolfner, 1997; Park et al., 1998). Insect seminal fluid proteins and peptides are transferred to action sites within and outside the female reproductive tract. The fluids lead to numerous responses in females (Soller et al., 1997; Chapman et al., 2003; Liu and Kubli, 2003; Clifton et al., 2014). Silencing NISPF-L in unmated males did not affect JH III titers compared to controls, although the outcome was otherwise in mated males, with increased JH III 


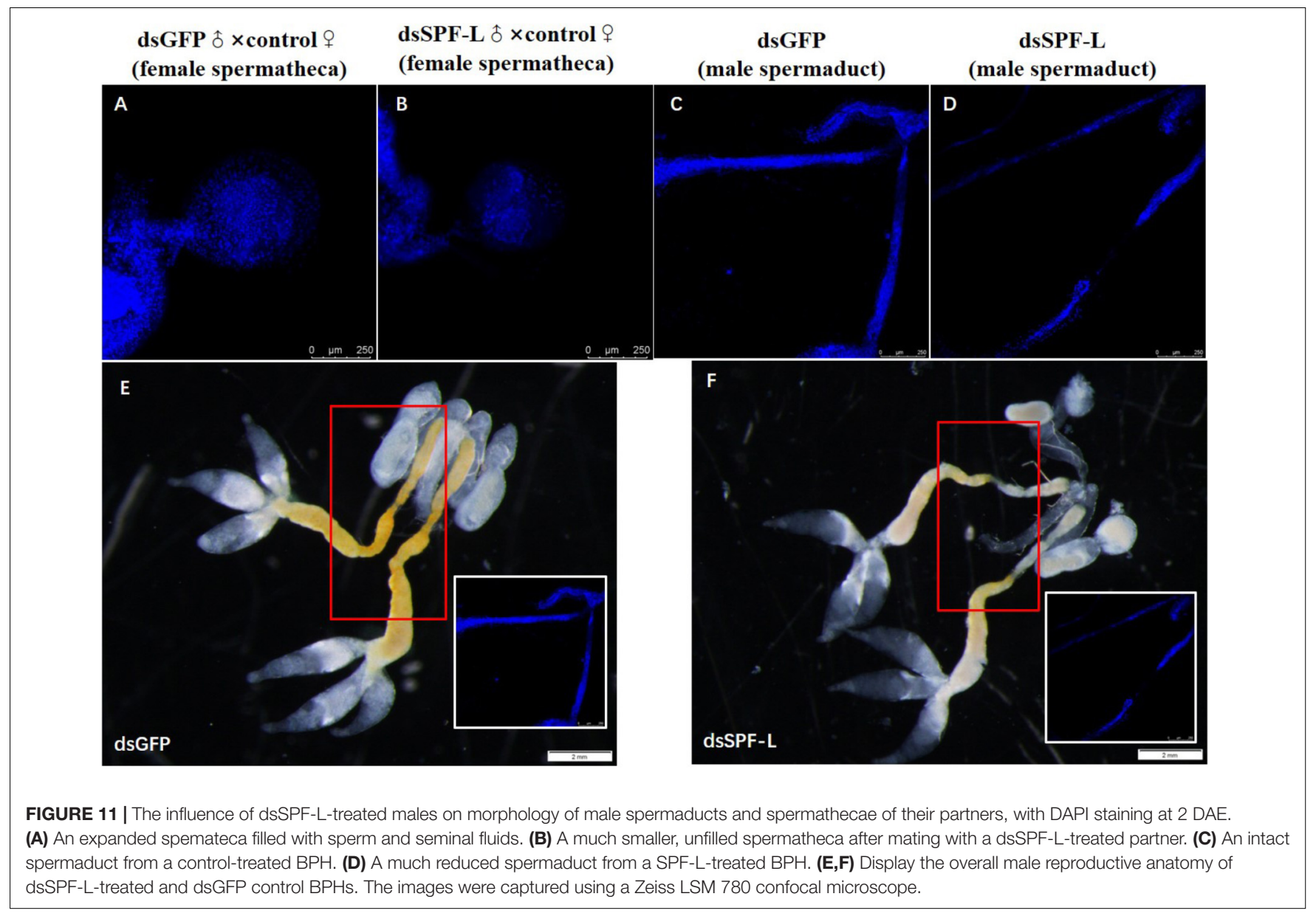

TABLE 2 | Mating with dsSPF-L-treated males led to reduced egg laying, hatch rates, and PGI.

\begin{tabular}{|c|c|c|c|c|}
\hline Treatments & Number of offspring & Hatching rate & Gender ratio & $\mathrm{PGI}\left(N_{1} / N_{0}\right)$ \\
\hline dsGFPor $\times$ Controlo & $782.4 \pm 35.3 a$ & $0.89 \pm 0.01 a$ & $1.11 \pm 0.03 a$ & 97.8 \\
\hline dsSepo $\times$ Controlo & $442.8 \pm 14.7 b$ & $0.64 \pm 0.03 b$ & $1.10 \pm 0.04 a$ & 55.4 \\
\hline
\end{tabular}

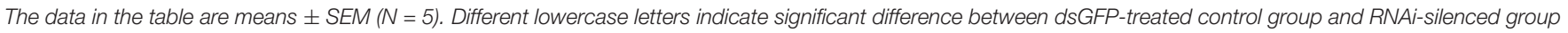
at $P<0.05$ by Student's t-test. $N O=4$ pairs (female $\times$ male).

titer and reduced JH III in mated females. We inferred that silencing NlSPF-L might influence the mating frequency or times of males, resulting in lower sperm, JH III, and other transmitted nutritional substances, which may be partially attributed to the deformed and thin vas efferens. Hyalophora cecropia and Heliothis virescens transfer $\mathrm{JH}$ to females within seminal fluid, which results in increased $\mathrm{JH}$ titers in female hemolymph and promotes egg development as well as maturity (Wolfner, 1997; Park et al., 1998). Furthermore, we found silencing SPF-L led to down-regulation of $N l V g$ expression, and did not influence $N l V g R$ expression at 2 DAE. Although $N l V g R$ expression level was not influenced by dsSPF-L treatments, suggesting $N l V g$ and $N l V g R$ expression time might not reach a consensus. Tufail et al. (2010) reported Vg expression was regulated by JH in N. lugens, and silencing $N l V g R$ significantly inhibited ovarian development and accumulation of the $\mathrm{Vg}$ protein in the hemolymp (Tufail et al., 2010), and the mRNA and protein levels of $N l V g$ were up-regulated by JHIII (Lu et al., 2015). These evidences indicated that RNAi-SPF-L indirectly influenced JH III titer, resulting in down-regulation of $V g$ expression and $\mathrm{Vg}$ protein synthesis, and inhibiting ovarian development and fecundity. The previous results demonstrated that MAGPs of TZP-treated males significantly increased compared to untreated controls along with JH III titer (Ge et al., 2010b; Wang et al., 2010), thus stimulating female fecundity via mating (Wang et al., 2010). Zhuo et al. (2018) Silencing the BPH doublesex gene, Nlds $x^{M}$, resulted in pseudofemales, with malformed sexual anatomy that led to male infertility. Here, by nuclear staining analysis of female spermatheca we found that mating with dsSPF-L-treated males led to reduced spermathecal sizes, probably due to lack of sperm. The present study provided the valuable information that SPF-L has potential as a novel target gene for control of phloem-feeding 
pest insects. In D. melanogaster, selenoprotein biosynthesis was activated by the Ras/MAPK signaling pathway by the redox (Morey et al., 2001). However, how SPF-L signaling pathway regulates reproduction in $N$. lugens needs further research.

\section{DATA AVAILABILITY STATEMENT}

All datasets generated for this study are included in the manuscript/supplementary files.

\section{AUTHOR CONTRIBUTIONS}

LG designed the research. YZ, HG, QW, ZZ, and SZ conducted the experiments. LG wrote the first draft

\section{REFERENCES}

Avila, F. W., Sirot, L. K., LaFlamme, B. A., Rubinstein, C. D., and Wolfner, M. F. (2011). Insect seminal fluid proteins: identification and function. Annu. Rev. Entomol. 56, 21-40. doi: 10.1146/annurev-ento-127090144823

Bradford, M. M. (1976). A rapid and sensitive method for the quantitation of microgram quantities of protein utilizing the principle of protein-dye binding. Anal. Biochem. 72, 248-254. doi: 10.1016/0003-2697(76)90527-3

Brown, D. G., and Burk, R. F. (1973). Selenium retention in tissues and sperm of rats fed a Torula yeast diet. J. Nutr. 103, 102-108. doi: 10.1093/jn/ 103.102

Chapman, T., Bangham, J., Vinti, G., Seifried, B., Lung, O., Wolfner, M. F., et al. (2003). The sex peptide of Drosophila melanogaster: female post-mating responses analyzed by using RNA interference. Proc. Natl. Acad. Sci. U.S.A. 100, 9923-9928. doi: 10.1073/pnas.1631635100

Chen, J., Zhang, D., Yao, Q., Zhang, J., Dong, X., and Tian, H. (2010). Feedingbased RNA interference of a trehalose phosphate synthase gene in the brown planthopper, Nilaparvata lugens. Insect Mol. Biol. 19, 777-786. doi: 10.1111/j. 1365-2538.2010.01038.X

Cheng, J. (2014). "Rice planthopper in the past half century in China," in Rice planthoppers, eds K. L. Heong, J. Cheng, and M. M. Escalada, (Dordrecht: Springer Netherlands), 1-32. doi: 10.1007/978-94-017-9535-7_1

Clifton, M. E., Correa, S., Riveera-Perez, C., Nouzova, M., and Noriega, F. G. (2014). Male Aedes aegypti mosquitoes use JH III transferred during copulation to influence previtellogenic ovary physiology and effected the reproductive output of female mosquitoes. J. Insect Physiol. 64, 40-47. doi: 10.1016/J.jinsphy. 2014.03.006

Ferguson, A. D., Labunskyy, V. M., Fomenko, D. E., Arac, D., Chelliah, Y., Amezcua, C. A., et al. (2006). NMR structures of the selenoproteons Sep 15 and SeIM reveal redox activity of a new thioredoxin-like family. J. Biol. Chem. 281, 3536-3543. doi: 10.1014/jbc.M511386200

Fu, Q., Zhang, Z. T., Lai, F. X., and Hu, C. (2001). A chemically defined diet enables the continuous rearing of the brown planthopper, Nilaparvata lugens (Stål). Appl. Entomol. Zool. 36, 1111-1116. doi: 10.1303/aez.2001.111

Fujisawa, M., Yamanaka, K., Tanaka, H., Tanaka, H., Okada, H., Arakawa, S., et al. (2001). Expression endothelial nitric oxide synthase in the Sertoli cells of men with infertility of various causes. BJU Int. 87, 85-88. doi: 10.1046/j.1464-410x. 2001.00986.x

Garcia-Gonzalez, F., and Simmons, L. W. (2005). Sperm viability matters in insect sperm competition. Curr. Biol. 15, 271-275.1.

Ge, L. Q., Gu, H. T., Huang, B., Song, Q. S., Stanley, D., Liu, F., et al. (2017a). An adenylyl cyclase like-9 gene (NlAC9) influences growth and fecundity in the brown planthopper, Nilaparvata lugens (Stå)(Hemiptera: Delphacidae). PLoS One 12:e0189214. doi: 10.1371/journal.pone.0189214 of the manuscript. QS and DS revised the final draft of the manuscript.

\section{FUNDING}

This work was co-financed by grants in aid from the National Key R\&D Program of China (2017YFD0200400), the National Natural Science Foundation of China (31872283), and the Natural Science Foundation of Jiangsu Province (BK20171283), the Jiangsu Agricultural Scientific Sel-inovation Fund, China (BE2018355).

\section{ACKNOWLEDGMENTS}

We thank Prof. Xu HaiJun of Zhejiang University for providing the Nilaparvata lugens anti-rabbit Vg.

Ge, L. Q., Xia, T., Huang, B., Gu, H. T., Song, Q. S., Yang, G. Q., et al. (2017b). PHF7, a novel male gene influences female fecundity and population growth in Nilaparvata lugens Stål (Hemiptera: Delphacidae). Sci. Rep. 7:11611. doi: 10.1038/S41598-017-11524-2

Ge, L. Q., Gu, H. T., Li, X., Zheng, S., Zhou, Z., Miao, H., et al. (2019). Silencing of triazopos-induced Hexokinase-1-like reduces fecundity in Nilaparvata lugens (Stål) (Hemiptera: Delphacidae). Pestic. Biochem. Phsyiol. 153, 176-184. doi: 10.1016/j.pestbp.2018.11.016

Ge, L. Q., Wang, L. P., Zhao, K. F., Wu, J. C., and Huang, L. J. (2010a). Mating pair combinations of insecticide-treated male and female Nilaparvata lugens Stål (Hemiptera: Delphacidae) planthoppers influence protein content in the male accessory glands (MAGs) and vitellin content in both fat bodies and ovaries of adult females. Pestic. Biochem. Physiol. 98, 278-288. doi: 10.1016/j.pestbp.2010. 06.019

Ge, L. Q., Wu, J. C., Zhao, K. F., Chen, Y., and Yang, G. (2010b). Induction of Nlvg and suppression of Nljhe gene expression in Nilaparvata lugens (Stål) (Hemiptera: Delphacidae) adult females and males exposed to two insecticides. Pestic. Biochem. Physiol. 98, 269-277. doi: 10.1016/j.pestbp.2010.06.018

Gillott, C. (2003). Male accessory gland secretions: modulators of female reproductive physiology and behavior. Ann. Rev. Entomol. 48, 163-184. doi: 10.1146/annurev.ento.48.091801.112657

Gladyshev, V. N., Jeang, K. T., Wootton, J. C., and Hatfield, D. L. A. (1998). A new human selenium-containing protein. Purification, characterization, and cDNA sequence. J. Biol. Chem. 27, 8910-8915. doi: 10.1074/jbc.273.15.8910

Jeanmougin, F., Thompson, J. D., Gouy, M., Higgins, D. G., and Gibson, T. J. (1998). Multiple sequence alignment with Clustal X. Trends Biochem. Sci. 23, 403-405. doi: 10.1016/S0968-0004(98)01285-7

LaFlamme, B. A., and Wolfner, M. F. (2013). Identification and function of proteolysis regulators in seminal fluid. Mol. Reprod. Dev. 80, 80-101. doi: 10. $12 / \mathrm{mrd} .22130$

Liu, H., and Kubli, E. (2003). Sex-peptide is the molecular basis of the sperm effect in Drosophila melanogaster. Proc Natl Acad. Sci U.S.A. 100, 9929-9933. doi: $10.1073 /$ pnas. 1631700100

Livak, K. J., and Schmittgen, T. D. (2001). Analysis of relative gene expression data using real-time quantitative PCR and the 2- $\triangle \triangle \mathrm{CT}$ method. Methods 25, 402-408. doi: 10.1006/meth.2001.1262

Loher, W., Ganjian, I., Kubo, I., Stanley, D., and Tobe, S. (1981). Prostaglandins: their role in egg-laying in the cricket Teleogryllus commodus. Proc. Natl. Acad. Sci. U.S.A. 78, 7835-7838. doi: 10.1073/pnas.78.12.7835

Long, Y., Hou, M. L., Yang, X., and Shi, B. K. (2010). Effects of delayed mating on reproduction in brachypterous females of brown planthopper, Nilaparvata lugens. Plant Prot. 6, 36-39. doi: 10.3969/j.issn.0529-542.2010.06.008

Lu, K., Chen, X., Liu, W. T., and Zhou, Q. (2016). TOR-pathway-mediated juvenile hormone synthesis regulates nutrient-dependent female reproduction in Nilaparvata lugens (Stål). Int. J. Mol. Sci. 17:438. doi: 10.3390/ijms17040438 
Lu, K., Shu, Y. H., Zhou, J. L., Zhang, X. Y., Zhang, X. Y., Chen, M. X., et al. (2015). Molecular characterization and RNA interference analysis of vitellogenin receptor from Nilaparvata lugens (Stål). J. Insect Physiol. 73, 20-29. doi: 10.1016/j.jinsphys.2015.01.007

Martin-Romero, F. J., Kryukov, G. V., Lobanov, A. V., Carlson, B. A., Lee, B. J., Gladyshev, V. N., et al. (2001). Selenium metabolism in Drosophila: selenoproteins, selenoprotein mRNA expression, fertility, and mortality. J. Biol. Chem. 276, 29798-29804. doi: 10.1074/jbc.M100422200

Michaelis, M., Gralla, O., Behrends, T., Scharpf, M., Endermann, T., Rijntjes, E., et al. (2014). Selenoprotein P in seminal fluid is a novel biomarker of sperm quality. Biochem. Biophys. Res. Commun. 443, 905-910. doi: 10.1016/j.bbrc. 2013.12.067

Morey, M., Serras, F., Baguà, J., Hafen, E., and Corominas, M. (2001). Modulation of the Ras/MAPK signaling pathway by the redox function of selenoproteins in Drosophila melanogaster. Dev. Biol. 238, 145-156. doi: 10.1006/dbio.2001. 0389

Park, Y. I., Ramaswamy, S. B., and Srinvasan, A. (1998). Spermatophore formation and regulation of egg maturation and oviposition in female Heliothis viresecens by the male. J. Insect Physiol. 44, 903-908. doi: 10.1016/s0022-1910(98) 00065-1

Poinani, A. (2006). Complexity of seminal fluid: a review. Behav. Ecol. Sociobiol. 60, 289-310. doi: 10.1007/s00265-006-0178-0

Ravi Ram, K., and Wolfner, M. F. (2007). Seminal influences: Drosophila A and the molecular interplay between males and females during reproduction. Intergr. Comp. Biol. 47, 427-445. doi: 10.1093/icb/icm046

Ravi Ram, K., and Wolfner, M. F. (2009). A network of interactions among seminal proteins underlies the long-term post-mating response in Drosophila. Proc. Natl. Acad. Sci. U.S.A. 106, 15384-15389. doi: 10.1073/pnas.09029 23106

Schneider, C. A., Rasband, W. S., and Eliceiri, K. W. (2012). NIH Image to ImageJ: 25 years of image analysis. Nat. Methods 9, 671-675. doi: 10.1038/nmeth.2089

Simmons, L. (2001). Sperm Competition and its Evolutionary Consequences in the Insects. Princeton, NJ: Princeton University Press.

Soller, M., Bownes, M., and Kubli, E. (1997). Mating and sex peptide stimulate the accumulation of yolk in ooccytes of Drosophila melanogaster. Eur. J. Biochem. 243, 732-738. doi: 10.1111/j.1432-1033.1997.00732.X

Stadtman, T. C. (1996). Selenocysteine. Annu. Rev. Biochem. 65, 83-100. doi: 10.1002/9780470015902.a0000688.pub3

Tamura, K., Peterson, D., Peterson, N., Stecher, G., Nei, M., and Kumar, S. (2011). MEGA5: molecular evolutionary genetics analysis using maximum likelihood, evolutionary genetics analysis using maximum likelihood, evolutionary distance, and maximum parsimony methods. Mol. Biol. Evol. 28, 2731-2739. doi: 10.1093/molbev/msr121

Tang, Q. Y., and Feng, M. G. (2002). “Analysis of variance,” in DPS Data Processing System for Practical Statistics-4, eds Q. Y. Tang, and M. G. Feng, (Beijing: Scientific Press), 47-71.

Tufail, M., Naeemullah, M., Elmogy, M., Sharma, P. N., Takeda, M., and Nakamura, C. (2010). Molecular cloning, transcriptional regulation, and differential expression profiling of vitellogenin in two wing-morphs of the brown planthopper, Nilaparvata lugens Stål (Hemiptera: Delphacidae). Insect Mol. Biol. 19, 787-798. doi: 10.1111/j.1365-2583.2010.01035.x

Wang, L. P., Shen, J., Ge, L. Q., Wu, J. C., Yang, G. Q., and Jahn, G. C. (2010). Insecticide-induced increase in the protein content of male accessory glands and its effect on the fecundity of females in the brown planthopper Nilaparvata lugens Stål (Hemiptera: Delphacidae). Crop Prot. 29, 1280-1285. doi: 10.1016/j. cropro.2010.07.009

Wolfner, M. F. (1997). Tokens of love: functions and regulation of Drosophila male accessory gland products. Insect Biochem. Mol. Biol. 27, 179-192. doi: 10.1016/s0965-1748(96)00084-7

Yu, B., Li, D. T., Lu, J. B., Zhang, W. X., and Zhang, C. X. (2016). Seminal fluid protein genes of the brown planthopper, Nilaparvata lugens. BMC Genomics 17:654. doi: 10.1186/s12864-016-3013-7

Zhang, H., and Zheng, R. L. (1996). Possible role of nitric oxide on fertile and asthenozoospermic infertile human sperm functions. Free Radic. Res. 25, 347-354. doi: 10.3109/10715769609149057

Zhuo, J. C., Hu, Q. L., Zhang, H. H., Zhang, M. Q., Jo, S. B., and Zhang, C. X. (2018). Identification and functional analysis of the doublesex gene in the sexual development of a hemimetabolous insect, the brown planthopper. Insect Biochem. Mol. Biol. 102, 31-42. doi: 10.1016/j.ibmb.2018.09.007 doi: 10.1016/j. ibmb.2018.09.007

Conflict of Interest: The authors declare that the research was conducted in the absence of any commercial or financial relationships that could be construed as a potential conflict of interest.

Copyright (C) 2019 Ge, Zhou, Gu, Wu, Zhou, Zheng, Stanley and Song. This is an open-access article distributed under the terms of the Creative Commons Attribution License (CC BY). The use, distribution or reproduction in other forums is permitted, provided the original author(s) and the copyright owner(s) are credited and that the original publication in this journal is cited, in accordance with accepted academic practice. No use, distribution or reproduction is permitted which does not comply with these terms. 